\title{
Electrical and mechanical behaviour of metal thin films with deformation-induced cracks predicted by computational homogenisation
}

\author{
T. Kaiser $($ ) M. J. Cordill(D) • \\ C. Kirchlechner $\mathbb{D}$ - A. Menzel $(\mathbb{D}$
}

Received: 12 January 2021 / Accepted: 22 July 2021 / Published online: 5 October 2021

(C) The Author(s) 2021

\begin{abstract}
Motivated by advances in flexible electronic technologies and by the endeavour to develop non-destructive testing methods, this article analyses the capability of computational multiscale formulations to predict the influence of microscale cracks on effective macroscopic electrical and mechanical material properties. To this end, thin metal films under mechanical load are experimentally analysed by using in-situ confocal laser scanning microscopy (CLSM) and in-situ four point probe resistance measurements. Image processing techniques are then used to generate representative volume elements from the laser intensity images. These discrete representations of the crack pattern at the microscale serve as the basis for the calcu-
\end{abstract}

T. Kaiser $(\bowtie) \cdot$ A. Menzel

Institute of Mechanics, TU Dortmund University, Leonhard-Euler-Str. 5, 44227 Dortmund, Germany

e-mail: tobias.kaiser@tu-dortmund.de

e-mail: andreas.menzel@udo.edu

M. J. Cordill

Erich Schmid Institute of Materials Science, Austrian

Academy of Sciences, Jahnstrasse 12, 8700 Leoben,

Austria

e-mail: megan.cordill@oeaw.ac.at

C. Kirchlechner

Institute for Applied Materials, Karlsruher Institute of

Technology, Hermann von Helmholtz Platz 1, 76344

Eggenstein-Leopoldshafen, Germany

e-mail: christoph.kirchlechner@kit.edu

A. Menzel

Division of Solid Mechanics, Lund University, P.O. Box

118, 22100 Lund, Sweden

e-mail: andreas.menzel@solid.lth.se lation of effective macroscopic electrical conductivity and mechanical stiffness tensors by means of computational homogenisation approaches. A comparison of simulation results with experimental electrical resistance measurements and a detailed study of fundamental numerical properties demonstrates the applicability of the proposed approach. In particular, the (numerical) errors that are induced by the representative volume element size and by the finite element discretisation are studied, and the influence of the filter that is used in the generation process of the representative volume element is analysed.

Keywords Computational multiscale simulations . Computational homogenisation · Scale-bridging · Electrical resistance $\cdot$ Microcracking $\cdot$ Anisotropic conductivity $\cdot$ Heterogeneous microstructures

\section{Introduction}

Flexible electronics need to be stretchable and foldable. However, cyclic loading causes more mechanical damage in terms of crack nucleation and growth than monotonic stretching or flex to connect loading (Glushko et al. 2017; Kreiml et al. 2019). The mechanical damage that forms leads to electrical failure due to the formation of plastic localisation zones (necks), extrusions, and eventually through thickness cracks (TTCs). Through thickness cracks are the leading cause of electrical failure in flexible thin 
film material systems. What is still missing in the research community is the direct correlation between mechanical damage and the electrical failure. Vice versa, changes in the measured electrical resistance can be interpreted as a fingerprint of damage inside the body under consideration and are thus valuable for the development of non-destructive testing methods.

From an experimental point of view, the main challenge to achieve reliability models is to properly identify TTCs and necks, especially in ductile conductive films and lines. In the experiments, monotonic and cyclic straining methods as well as cyclic bending tests are used to induce microscale cracks in the thin films, and the associated changes in electrical resistance are analysed by four point probe (4PP) measurements (Gruber et al. 2009; Lu et al. 2010; Lambricht et al. 2013; Sim et al. 2013; Glushko and Cordill 2016; Kreiml et al. 2019). In addition, characteristic properties like the crack onset strain (COS) are determined, and the evolution of the mechanical damage in terms of TTCs and localised necking is studied with optical microscopy, scanning electron microscopy (SEM), atomic force microscopy (AFM) or confocal laser scanning microscopy (CLSM) methods (Renault et al. 2003; Leterrier et al. 2004; Cordill et al. 2010; Jin et al. 2011; Cordill et al. 2015, 2016; Etiemble et al. 2019; Cahn et al. 2020). For brittle film systems, SEM and optical observation methods are adequate because only TTCs form, and when combined with in-situ 4PP measurements, these are quite precise at determining the COS and crack spacing or density evolution as a function of applied strain or cycle. However, for ductile films three-dimensional surface imaging methods, such as AFM or CLSM, are necessary in order to properly identify TTCs and necks (Cordill and Marx 2013a, b; Cordill et al. 2015; Berger et al. 2016). More specifically speaking, TTCs can be distinguished from localised necks, either due to the difference in depth in the height images or due to the difference in intensity in the CLSM laser intensity images. A current drawback when using height and intensity images is the time intensive, and somewhat subjective, data analysis with low statistics. In particular, the current state-of-the-art data analysis method only extracts cross-section or surface profiles, similar to evaluating the linear crack density in brittle films, and may only capture a small portion of the mechanical damage in the whole image. Faster (automated) imaging analysis techniques that can identify TTCs and necks in a whole image are thus required. Moreover, the combined electro-mechanical behaviour of metal thin films is not yet understood to such an extent that reliable lifetime predictions can be developed. Against this background and in an attempt to bridge the gap between materials science and computational mechanics, the suitability of advanced multiscale simulation techniques that take detailed microscale information into account and that may contribute to an understanding of the complex electro-mechanically coupled behaviour of metal thin films is studied in this contribution.

Functional material properties that are observable at the macroscale are governed by the underlying microstructure and, possibly, crack pattern induced for example due to mechanical (Glushko and Cordill 2016) or thermo-mechanical loads (Moser et al. 2019). Extending classic phenomenological material models, distinct features at the microscale are resolved in computational multiscale formulations. These may include, but are not limited to, different phases, grain boundaries, inclusions and cracks. To this end, the evaluation of constitutive relations is substituted by an additional finite element calculation of the underlying microstructure. The boundary conditions are prescribed based on the macroscopic material state and effective macroscopic quantities, e.g. stresses and electric current densities, follow from the homogenisation of their microscopic analogues. Computational multiscale formulations are meanwhile well-established and applications of first-order $\mathrm{FE}^{2}$-based approaches to purely mechanical problems are for example discussed in Miehe et al. (1999), Feyel and Chaboche (2000), Kouznetsova et al. (2001), Ricker et al. (2010), Coenen et al. (2012a,b), and Gu et al. (2017). More elaborated, second-order computational homogenisation methods that additionally account for the gradient of the macroscopic deformation tensor are studied in, e.g., Kouznetsova et al. (2002, 2004). Moreover, the extension to thermo-mechanically coupled problems is presented in Özdemir et al. (2008), Temizer and Wriggers (2011), Sengupta et al. (2012), Temizer (2016), Berthelsen et al. (2017), Berthelsen and Menzel (2019) and electro-mechanically coupled multiscale formulations of dielectric materials are elaborated in Schröder (2009), Khalaquzzaman et al. (2012), and Keip et al. (2014). Based on, and as an extension of these works, an electro-mechanically coupled multiscale formulation 
for electrical conductors has recently been proposed in Kaiser and Menzel (2021). In principle, this formulation allows the studying of the influence of microscale deformation processes and of microscale cracks on the effective electrical and mechanical material properties at the macroscale. To the authors' best knowledge, this promising approach has not been applied before to analyse the influence of microscale cracks (in metal thin films) on effective electrical properties that are experimentally observed at the macrosale. The present work thus contributes to the fundamental research question: Can the experimentally recorded changes in the electrical properties of metal thin films be related to the experimentally recorded microscale cracks by accounting for CLSM laser intensity information in simplified twodimensional representative volume elements (RVEs)? In this regard, the influence of the non-trivial RVEgeneration process and of the RVE-size on the predicted macroscopic electrical conductivity tensor will be studied in detail. In addition, effective mechanical material parameters will be calculated for the deformation states studied. Although a direct comparison of the latter with experimental results is not feasible for the metal thin films that are in the focus of the present contribution, interesting differences in the evolution of the mechanical and the electrical material parameters are observed which are important for the development of non-destructive electric potential-based testing methods as elaborated in, e.g., Tada et al. (1996, 1997), Tada (2006). Furthermore, it is noted that the cracks are geometrically resolved for each deformation state based on the experimental CLSM laser intensity images, with the modeling of crack initiation and crack propagation not being in the focus of the present analysis. As a proof of concept-type contribution this work thus establishes a basis for future developments.

The article is organised as follows: Experimental investigations on metal thin films in terms of in-situ CLSM and 4PP resistance measurements are discussed in Sect. 2. These motivate the (small-deformation) computational homogenisation scheme proposed in Sect. 3. The latter is applied in Sect. 4 to calculate effective macroscopic conductivity and stiffness tensors for experimentally recorded micrographs that are compared with experiments in Sect. 5. Finally, a brief summary of the findings is presented in Sect. 6.

\subsection{Notation}

In this contribution, single tensor contractions are used in the sense $[\boldsymbol{\alpha} \otimes \boldsymbol{\beta}] \cdot[\boldsymbol{\gamma} \otimes \boldsymbol{\delta}]=[\boldsymbol{\beta} \cdot \boldsymbol{\gamma}][\boldsymbol{\alpha} \otimes \boldsymbol{\delta}]$ and double tensor contractions are given by $[\boldsymbol{\alpha} \otimes \boldsymbol{\beta}]$ : $[\boldsymbol{\gamma} \otimes \delta]=[\boldsymbol{\alpha} \cdot \boldsymbol{\gamma}][\boldsymbol{\beta} \cdot \boldsymbol{\delta}]$ with $\boldsymbol{\alpha}, \boldsymbol{\beta}, \boldsymbol{\gamma}$ and $\delta$ denoting arbitrary first-order tensors. In addition to standard dyadic products that are indicated by $\otimes$, the generalised tensor products $[\alpha \otimes \beta] \bar{\beta}[\boldsymbol{\gamma} \otimes \delta]=[\alpha \otimes \gamma] \otimes$ $[\boldsymbol{\beta} \otimes \delta]$ and $[\boldsymbol{\alpha} \otimes \boldsymbol{\beta}] \otimes[\boldsymbol{\gamma} \otimes \delta]=[\boldsymbol{\alpha} \otimes \boldsymbol{\gamma}] \otimes[\boldsymbol{\delta} \otimes \boldsymbol{\beta}]$ are introduced to allow for a compact notation. Moreover, the coefficient matrix of a second-order tensor $\boldsymbol{T}$ with respect to a Cartesian basis system $\boldsymbol{e}_{i}$ is indicated by $[\boldsymbol{T}]_{i j}$ and frequently used to simplify the presentation of results. The respective tensor coefficients $T_{i j}$ follow from the projection of $\boldsymbol{T}$ onto the basis, i.e. $T_{i j}=\boldsymbol{e}_{i} \cdot \boldsymbol{T} \cdot \boldsymbol{e}_{j}$. Furthermore, gradient, divergence and curl operators are denoted by $\nabla \bullet$, by $\nabla \cdot \bullet$ and by $\nabla \times \bullet$, respectively, and are applied in the sense of rightgradient, right-divergence and right-curl operations.

\section{Experiments}

The present contribution is based on the experiments documented in Cordil et al. (2017) where a bilayer made of a $200 \mathrm{~nm} \mathrm{Cu}$ film with a $10 \mathrm{~nm} \mathrm{Cr}$ adhesion layer on a $50 \mu \mathrm{m}$ Upilex Polyimide substrate was cyclically strained and analysed by using in-situ resistance measurements and CLSM imaging. The cycling was as follows: 1 slow cycle (displacement rate $0.1 \mu \mathrm{m} \mathrm{s}^{-1}$ ), 10 fast cycles (displacement rate $10 \mu \mathrm{m} \mathrm{s}^{-1}$ ), 1 slow, 10 fast, 1 slow, 100 fast, 1 slow, 25 fast, and 1 slow. The slow cycles were used to measure the lattice strain in the $\mathrm{Cu}$ film by using in-situ $\mathrm{X}$-ray diffraction where slower displacement rates are necessary. For the in-situ electrical measurements, the relative resistance ratio, $R / R_{0}$, is used where $R_{0}$ is the initial resistance before straining (without mechanical damage or cracks) and where $R$ is the instantaneous measured resistance. The relative resistance ratio as a function of the load cycle number is depicted in Fig. 1f. At the end of each cycle or set of multiple cycles, CLSM imaging was performed on the surface of the sample in the unloaded condition. With CLSM, three images can be created, (i) an optical light image, (ii) a laser intensity image, and (iii) a quantitative height image. In this contribution, the laser intensity images, as exemplarily shown in Fig. 1a-e are further evaluated. For more details about the material 


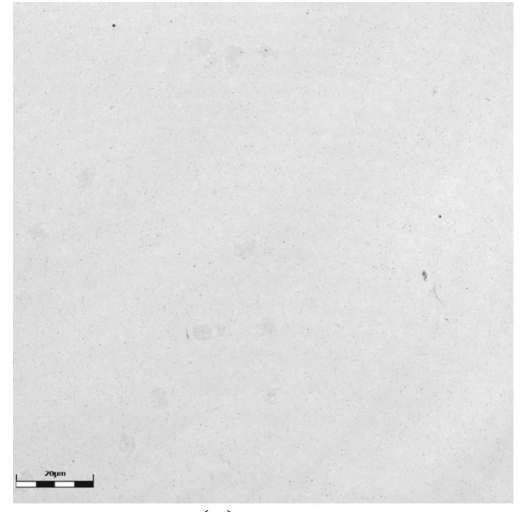

(a) cycle 1

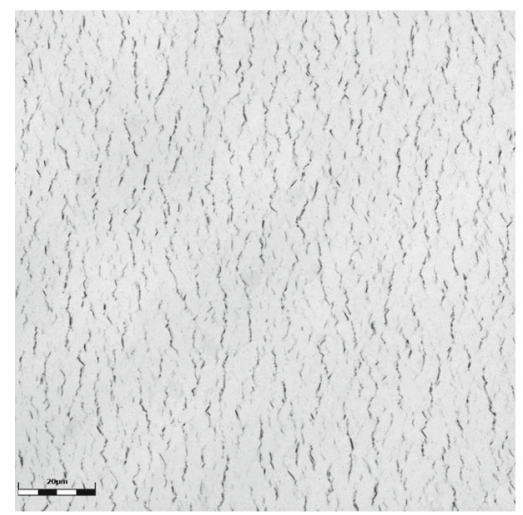

(d) cycle 123

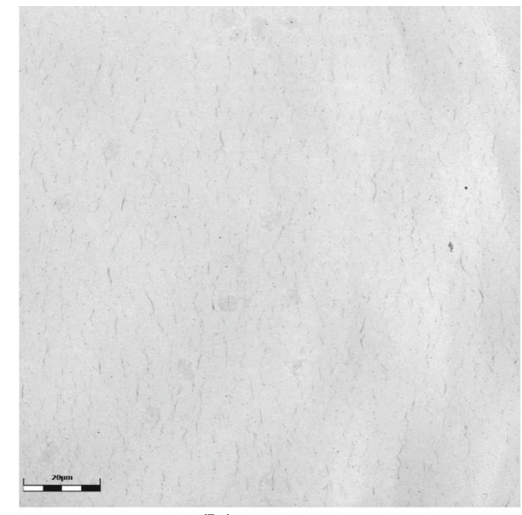

(b) cycle 11

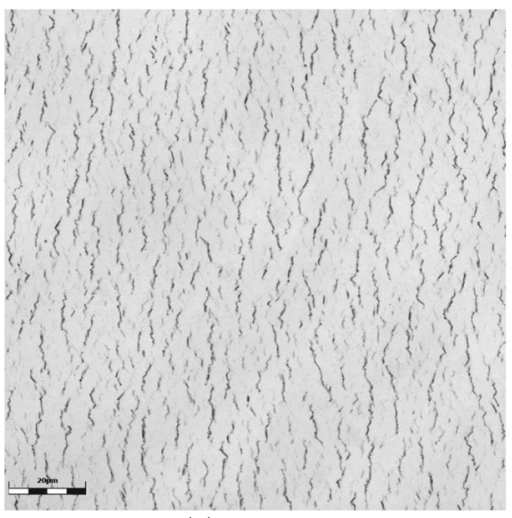

(e) cycle 148

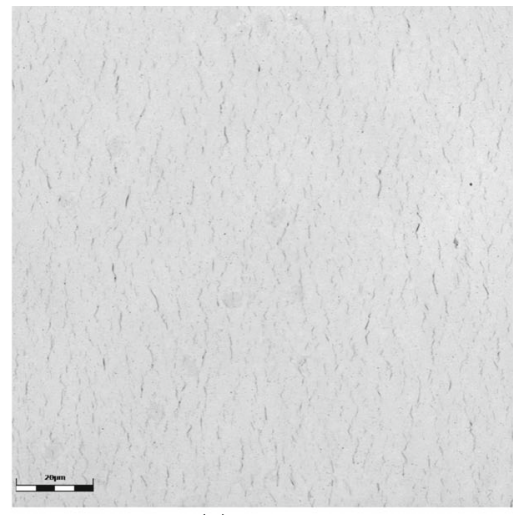

(c) cycle 22

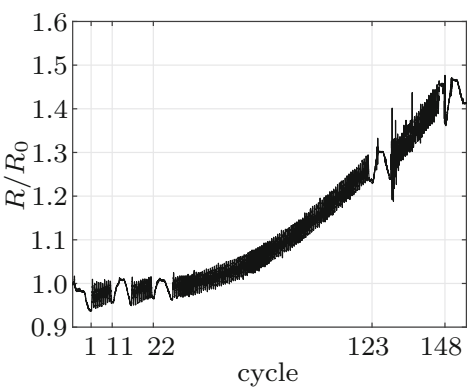

(f) relative resistance ratio
Fig. 1 a-e Laser intensity images for different deformation states of a $200 \mathrm{~nm} \mathrm{Cu}$ film with a $10 \mathrm{~nm} \mathrm{Cr}$ adhesion layer on a $50 \mu \mathrm{m}$ thick Upilex Polyimide substrate $(130 \mu \mathrm{m} \times 130 \mu \mathrm{m}$ micrographs). The tensile straining was parallel to the horizontal

system and the experiments, the reader is referred to Cordil et al. (2017).

\section{Calculation of effective conductivity and stiffness tensors}

This section briefly recapitulates the fundamentals of the multiscale computational homogenisation scheme proposed in Kaiser and Menzel (2021) that is used in the present contribution to predict effective macroscopic conductivity and elasticity tensors for the crack patterns presented in Sect. 2. In particular, the field equations are summarised in Sect. 3.1 and the constitutive models that are used at the microscale, respectively the computational homogenisation techniques that substitute the constitutive models at the macroscale, are presented in Sects. 3.2 and 3.3. direction and all scale bars are $20 \mu \mathrm{m}$. f Relative resistance ratio as a function of deformation parametrised in terms of the load cycle number

\subsection{Field equations}

Let the region that is occupied by the body under consideration at some reference time $t_{0} \in \mathbb{R}$ be denoted by $\mathcal{B} \subset \mathbb{R}^{3}$ and let material points be identified by their position in space $x \in \mathcal{B}$. The motion of the body is defined by the displacement field $\boldsymbol{u}(\boldsymbol{x}, t): \mathcal{B} \times \mathbb{R} \rightarrow$ $\mathbb{R}^{3}$ and the electric state is characterised in terms of the electric potential field $\phi(\boldsymbol{x}, t): \mathcal{B} \times \mathbb{R} \rightarrow \mathbb{R}$. Based on the primary field variables, the linearised strain tensor

$\boldsymbol{\varepsilon}=\frac{1}{2}\left[\nabla \boldsymbol{u}+[\nabla \boldsymbol{u}]^{\mathrm{t}}\right]$

and the electric field vector

$\boldsymbol{e}=-\nabla \phi$ 
are defined. In general, their energetic duals, i.e. stress tensor $\sigma$ and electric current density vector $\boldsymbol{j}$, are functions of $\boldsymbol{\varepsilon}, \boldsymbol{e}$ and, possibly, of temperature and some internal variables that have to fulfil the restrictions that are imposed by the second law of thermodynamics. Moreover, $\sigma$ and $j$ directly enter the respective field equations that characterise the mechanical and electrical problem. Focusing on quasi-static deformation processes and neglecting body forces $f$, the mechanical problem is characterised by the balance equation of linear momentum

$\nabla \cdot \sigma=\mathbf{0}$

and by the balance equation of angular momentum that reduces to the symmetry condition of the stress tensor

$\sigma=\sigma^{\mathrm{t}}$

Assuming quasi-stationary electric processes, the electrical problem is given in terms of the continuity equation for the electric current density

$\nabla \cdot \boldsymbol{j}=0$

and in terms of the Maxwell-Faraday equation with a vanishing induction term

$\nabla \times \boldsymbol{e}=\mathbf{0}$.

Following standard practise, the symmetry condition of the stress tensor (4) is fulfilled by the specific choice of the constitutive equations at the microscale and by the scale-bridging relation that relates the microscale stress tensor to its macroscopic analogue, cf. Sects. 3.2 and 3.3. Likewise, the Maxwell-Faraday equation (6) can a priori be fulfilled by the introduction of an electric potential according to (2). The set of balance equations thus reduces to a system of two (in general) coupled partial differential equations, i.e. (3) and (5), that is solved for the displacement field $\boldsymbol{u}$ and for the electric potential field $\phi$. Assuming linearised kinematics, the coupling may only be induced by the specific constitutive equations for the stress tensor and for the electric current density vector. In contrast, a coupling due to changes in geometry is additionally accounted for when finite deformations are considered, (Kaiser and Menzel 2021).
3.2 Constitutive relations at the macroscale

In computational multiscale simulations physical processes at different material length and time scales are considered, as schematically shown in Fig. 2. The principal idea of these approaches is to replace the phenomenological constitutive equations for the stress tensor $\sigma_{\mathrm{M}}$ and for the electric current density vector $\boldsymbol{j}_{\mathrm{M}}$ that are usually assumed at the macroscale by a computational homogenisation scheme that makes it possible to take detailed information of the underlying crack density and morphology into account. More specifically speaking, the cracks are geometrically resolved in representative volume elements which serve as generalised material models and which are assigned to each macroscopic material point $\boldsymbol{x}_{\mathrm{M}}$. Based on the macroscopic deformation state $\varepsilon_{M}$ and on the macroscopic electric field vector $\boldsymbol{e}_{\mathrm{M}}$, the RVE is subjected to electrical and mechanical boundary conditions that are in accordance with the extended Hill-Mandel conditions of the electro-mechanical problem under consideration. After the solution of the microscale boundary value problem, the macroscopic stress tensor

$\sigma_{\mathrm{M}}=\frac{1}{v_{\mathrm{m}}} \int_{\mathcal{B}_{\mathrm{m}}} \sigma_{\mathrm{m}} \mathrm{d} v$

and the macroscopic electric current density vector

$\boldsymbol{j}_{\mathrm{M}}=\frac{1}{v_{\mathrm{m}}} \int_{\mathcal{B}_{\mathrm{m}}} \boldsymbol{j}_{\mathrm{m}} \mathrm{d} v$

are calculated as volume averages of their microscopic counterparts, with $v_{\mathrm{m}}$ denoting the RVE-volume. In this regard, it is remarked that (7) and (8) can equivalently be expressed in terms of surface integrals which are evaluated in the present contribution subject to the assumption of stress-free internal boundaries that are electrically insulated (i.e., $\boldsymbol{\sigma}_{\mathrm{m}} \cdot \boldsymbol{n}=\mathbf{0}$ and $\boldsymbol{j}_{\mathrm{m}} \cdot \boldsymbol{n}=0$ are assumed at internal boundaries with outward unit normal vector $\boldsymbol{n}$ ). Moreover, the effective macroscopic mechanical tangent stiffness tensor

$E_{M}=\frac{\mathrm{d} \sigma_{M}}{\mathrm{~d} \varepsilon_{M}}$ 


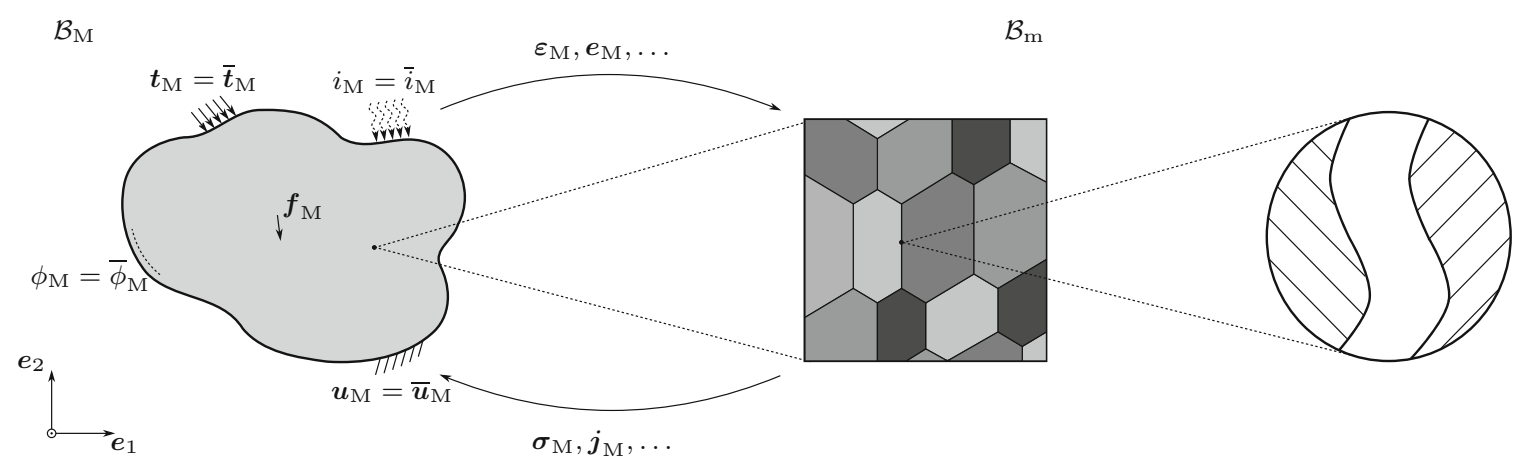

Fig. 2 First-order computational homogenisation scheme for electro-mechanical problems. At the microscale different material regions, representing for example different grains, are indi-

and the effective macroscopic electrical conductivity tensor

$\mathcal{S}_{\mathrm{M}}=\frac{\mathrm{d} \boldsymbol{j}_{\mathrm{M}}}{\mathrm{d} \boldsymbol{e}_{\mathrm{M}}}$

are extracted, as described in detail in Kaiser and Menzel (2021). These serve as a basis for the analysis of the influence of microstructural defects on effective macroscopic material properties that is aspired in this contribution.

\subsection{Constitutive relations at the microscale}

In experiments as well as in computational multiscale simulations, the underlying crack pattern and microstructure evolution manifest themselves in the effective macroscopic material behaviour. In this contribution, crack patterns at the microscale are geometrically resolved in representative volume elements that are prepared from experimental data by using image processing techniques as described in Sect. 4. Moreover, linear constitutive relations between the stresses and strains,

$\sigma_{\mathrm{m}}=\mathbf{E}_{\mathrm{m}}: \boldsymbol{\varepsilon}_{\mathrm{m}}$

respectively between the electric field and the electric current density

$\boldsymbol{j}_{\mathrm{m}}=\mathcal{S}_{\mathrm{m}} \cdot \boldsymbol{e}_{\mathrm{m}}$ cated (middle figure). In addition, cracks that are geometrically resolved are schematically depicted (right figure). Prescribed quantities are denoted with an overbar

are adopted at each material point of the microscale. Assuming isotropic mechanical and electrical properties, the elastic stiffness tensor takes the form

$\mathbf{E}_{\mathrm{m}}=\lambda \boldsymbol{I} \otimes \boldsymbol{I}+\mu[\boldsymbol{I} \bar{\otimes} \boldsymbol{I}+\boldsymbol{I} \underline{\otimes} \boldsymbol{I}]$

and the electrical conductivity tensor is given by

$\mathcal{S}_{\mathrm{m}}=\kappa \boldsymbol{I}$

The tensor $\boldsymbol{I}$ in the previous equations denotes the second-order identity tensor, $\lambda$ and $\mu$ denote the Lamé constants and $\kappa$ denotes the scalar-valued electrical conductivity of an idealised material. The material parameters that are used in the simulations to be discussed in Sect. 4 are summarised in Table 1 with

$$
\begin{aligned}
\lambda & =\frac{E v}{[1+v][1-2 v]}, \\
\mu & =\frac{E}{2[1+v]},
\end{aligned}
$$

and with $E$ denoting the Young's modulus and $v$ denoting the Poisson's ratio. Moreover, periodic-type boundary conditions are assumed and the two-dimensional simulations are carried out subject to the simplifying assumption of a plane strain setting. Additional simulation results that are based on the assumption of a plane stress setting are provided in Appendix A for the sake of comparison.

Remark 1 Care needs to be taken when interpreting the homogenised macroscale tangent stiffness tensor 
Table 1 Material parameters used in the simulations

\begin{tabular}{lll}
\hline$E$ & $v$ & $\kappa$ \\
\hline $130000 \mathrm{~N} / \mathrm{mm}^{2}$ & 0.35 & $58100 \mathrm{~A} /[\mathrm{Vmm}]$ \\
\hline
\end{tabular}

(9) since neither the irreversible processes at the crack tips nor contact conditions between two opposing sides of the cracks are accounted for in the simulations. Thus, the macroscale tangent stiffness tensor $\mathbf{E}_{\mathrm{M}}$ is to be interpreted as the purely elastic unloading stiffness of the current material state.

\section{Representative simulation results}

As a first step towards the multiscale analysis of metal thin films, fundamental (numerical) properties of the computational homogenisation scheme when applied to experimental data are evaluated in this section. More specifically speaking, the influence of different filter options in the generation process of the representative volume elements is investigated in Sect. 4.1. In a next step, the focus lies on the influence of the RVE-size. To this end, RVEs generated from $38 \mu \mathrm{m} \times 38 \mu \mathrm{m}$, $76 \mu \mathrm{m} \times 76 \mu \mathrm{m}$ and $114 \mu \mathrm{m} \times 114 \mu \mathrm{m}$ in-situ micrographs are analysed and the predicted macroscopic material properties are compared in Sect. 4.2. Finally, the influence of the finite element discretisation is studied in Sect. 4.3.

\subsection{Comparison of different filter options}

The computational multiscale simulations are based on representative volume elements that are generated from the experimental data provided in Fig. 1. In particular, image processing techniques are applied in order to identify individual cracks and the corresponding crack paths, with the focus of this section being on the influence of the filter value when processing the CLSM laser intensity images. The non-trivial distinction between TTCs and necking zones, which still allow for a (reduced) electric current, will additionally be subjected to a detailed analysis in Sect. 5.

The geometric data from the CLSM laser intensity images is converted into finite element meshes, which serve as the basis for the computational homogenisation scheme. Being more specific, greyscale gradients in $\boldsymbol{e}_{1}$-direction of the micrographs depicted in Fig. 1 are calculated in a first step, since the cracks preferably form in $\boldsymbol{e}_{2}$-direction due to the loading conditions. The $\boldsymbol{e}_{1}$ - and $\boldsymbol{e}_{2}$-direction are defined in accordance with Fig. 2 and are not indicated in Fig. 1 for the sake of clarity. In a second step, only pixels with negative greyscale gradient values that are higher than a factor $\gamma$ times the mean value of the negative greyscale gradients in $\boldsymbol{e}_{1}$-direction are considered to represent cracks. This condition can be stated as

$-\nabla \mathcal{G}_{i} \cdot \boldsymbol{e}_{1}>\gamma \frac{1}{\mathrm{n}_{\text {pix }}} \sum_{j=1}^{\mathrm{n}_{\text {pix }}} \max \left\{0,-\nabla \mathcal{G}_{j} \cdot \boldsymbol{e}_{1}\right\}$,

with $\mathcal{G} \bullet$ denoting the greyscale value of pixel $\bullet$ and with $\mathrm{n}_{\text {pix }}$ denoting the number of pixels in the image. Moreover, all geometric objects that have fewer than 15 pixels are removed from the binary image to reduce the noise that is for example induced by the speckles in Fig. 1a. In a third step, morphological operations are applied in order to simplify the crack geometries and, hence, to reduce the computational effort. In particular, the convex hull of the individual cracks is calculated before the cracks are thinned to lines, again. The crack paths are then approximated by straight lines connecting the end points of the original crack paths. In a final step, a representative volume element is prepared from the data, with the individual cracks being geometrically resolved and with $1 \%$ edge length being added to each side of the representative volume element in order to avoid that cracks intersect the domain boundaries.

In the following, the influence of the filter parameter $\gamma$ on the representative volume elements, and hence on the effective macroscopic material properties, is studied. To this end, three different values of the filter parameter $\gamma \in\{4.0,6.5,8.0\}$ are taken into account. The discrete microstructure representations that have been generated for the five deformation states depicted in Fig. 1, by using the procedure proposed at the beginning of this section, are provided in Figs. 3 and 4. Since the threshold for a pixel to be identified with a crack increases with increasing values of $\gamma$, both the number of cracks as well as the overall length of the cracks decreases with increasing values of $\gamma$. In order to quantify the previous observations, the number of cracks that have been identified for the three different values of the filter parameter and the five different deformation states are summarised in Table 2. Moreover, compar- 


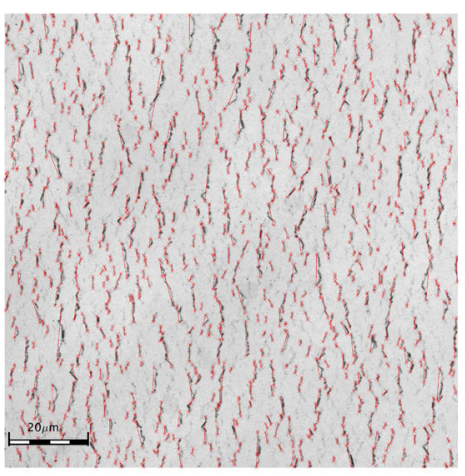

(a) cycle $123, \gamma=4.0$

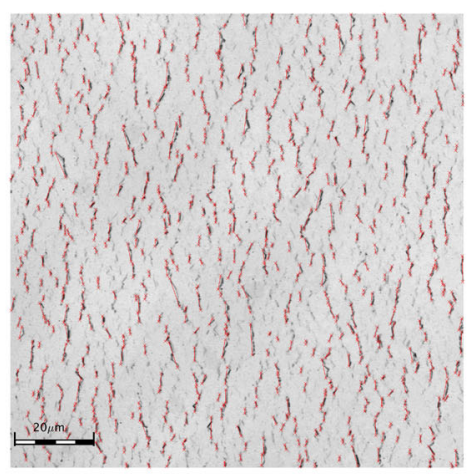

(b) cycle $123, \gamma=6.5$

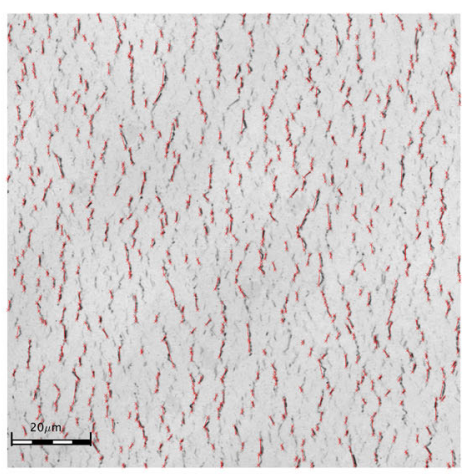

(c) cycle $123, \gamma=8.0$

Fig. 3 Comparison of identified cracks using three different filter options. Depicted are $114 \mu \mathrm{m} \times 114 \mu \mathrm{m}$ micrographs for $\gamma \in\{4.0,6.5,8.0\}$ with red lines indicating the cracks that have been identified

ing the discrete crack pattern representations of cycle 123 depicted in Fig. 3, the question arises as to whether regions where the greyscale gradient takes values such that they are identified with cracks for $\gamma=4.0$ but not for $\gamma=8.0$ are TTCs or rather plastic localisation zones that indicate the onset of cracking. This interesting question is subjected to a detailed analysis in Sect. 5 where additional data is taken into account.

The cracks that are accounted for at the microscale manifest themselves in the effective material properties at the macroscale. In this work, a particular focus lies on the electrical properties in terms of the conductivity tensor $\mathcal{S}_{\mathrm{M}}$ and on the mechanical properties in terms of the tangent stiffness tensor $\mathbf{E}_{\mathrm{M}}$. In this regard and with reference to the experimental (macroscopic) load-resistance curve depicted in Fig. If it is instructive to regard a simple conductor. Under the assumption of a quasi-homogeneous, uni-directional macroscopic electric current, the electrical resistance of the latter is given by

$R=\frac{1}{\kappa} \frac{L}{A}=R_{\text {spe }} R_{\text {geo }}, \quad R_{\text {spe }}=\frac{1}{\kappa}, \quad R_{\text {geo }}=\frac{L}{A}$

with $R_{\text {geo }}$ accounting for the (macroscopic) geometry of the conductor in terms of length $L$ and crosssectional area $A$, and with the electrical resistivity of the macroscopic material point $R_{\text {spe }}$ that results from a specific microstructure and crack pattern. In the present work, $\kappa$ or more generally speaking the respective coefficients of the macroscopic conductivity tensor $\mathcal{S}_{\mathrm{M}}$ are calculated by applying the computational homogenisation scheme discussed in Sect. 3.2 to the experimentally recorded crack patterns depicted in Fig. 1. For the $114 \mu \mathrm{m} \times 114 \mu \mathrm{m}$ micrographs and $\gamma=6.5$ these are given by

$$
\begin{aligned}
& {\left[\mathcal{S}_{\mathrm{M}}\right]_{i j}^{\mathrm{ref}} }=\left[\begin{array}{rr}
1.0000 & 0.0000 \\
0.0000 & 1.0000
\end{array}\right] \kappa \\
& {\left[\mathcal{S}_{\mathrm{M}}\right]_{i j}^{1}=\left[\begin{array}{rr}
0.9983 & -0.0001 \\
-0.0001 & 0.9999
\end{array}\right] \kappa } \\
& {\left[\mathcal{S}_{\mathrm{M}}\right]_{i j}^{11}=\left[\begin{array}{ll}
0.9754 & 0.0000 \\
0.0000 & 0.9990
\end{array}\right] \kappa } \\
& {\left[\mathcal{S}_{\mathrm{M}}\right]_{i j}^{22}=\left[\begin{array}{ll}
0.9305 & 0.0007 \\
0.0007 & 0.9972
\end{array}\right] \kappa } \\
& {\left[\mathcal{S}_{\mathrm{M}}\right]_{i j}^{123}=\left[\begin{array}{ll}
0.8173 & 0.0015 \\
0.0015 & 0.9912
\end{array}\right] \kappa } \\
& {\left[\mathcal{S}_{\mathrm{M}}\right]_{i j}^{148}=\left[\begin{array}{ll}
0.6753 & 0.0102 \\
0.0102 & 0.9893
\end{array}\right] \kappa }
\end{aligned}
$$

with superscript referring to the cycle number and $\mathcal{S}_{\mathrm{M}}^{\text {ref }}$ indicating the conductivity tensor of an idealised microstructure without cracks, i.e. the initial state before deformation. With regard to (18) it is observed that the 11-coefficient of the conductivity tensor gradually decreases with increasing deformation.

Moreover, a decrease in the 22-coefficient is observable which is, however, significantly smaller than the decrease in the 11-coefficient. Thus, the resulting effective properties at the macroscale are significantly anisotropic. This result can be explained by the pre- 


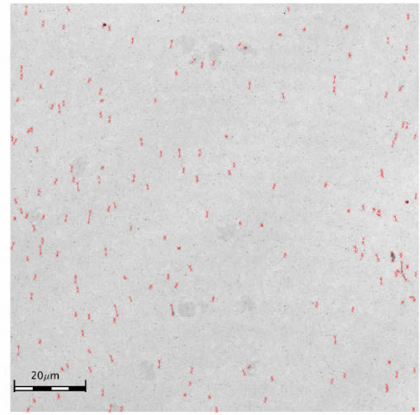

(a) cycle $1, \gamma=4.0$

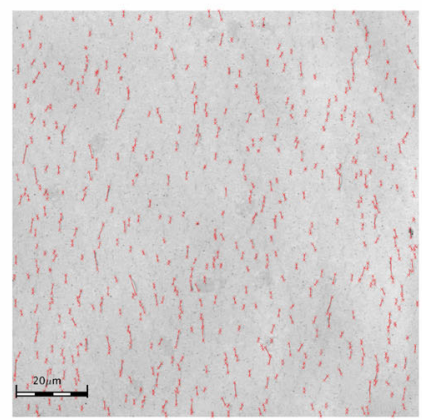

(d) cycle $11, \gamma=4.0$

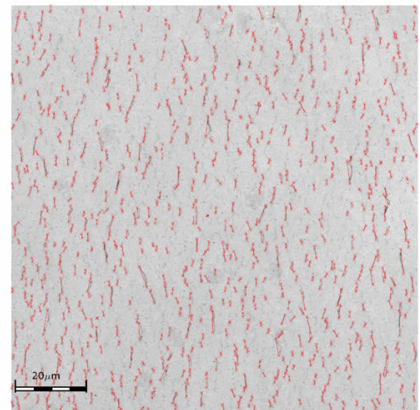

(g) cycle $22, \gamma=4.0$

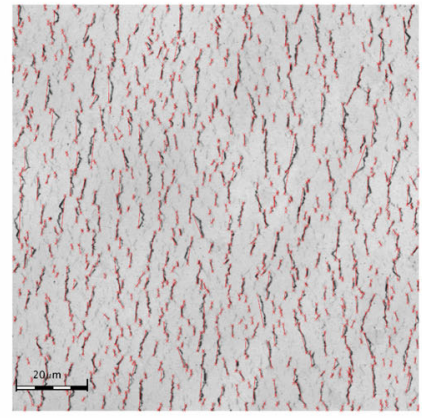

(j) cycle $148, \gamma=4.0$

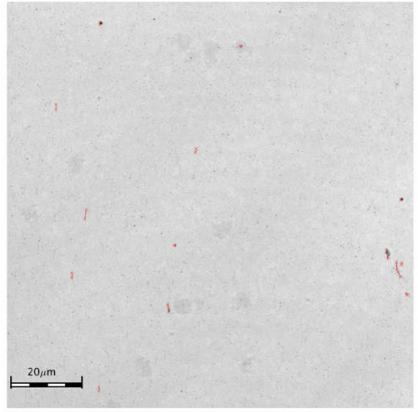

(b) cycle $1, \gamma=6.5$

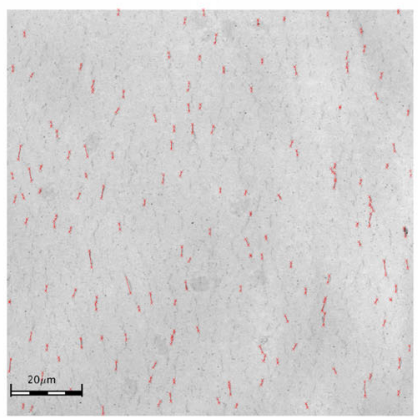

(e) cycle $11, \gamma=6.5$

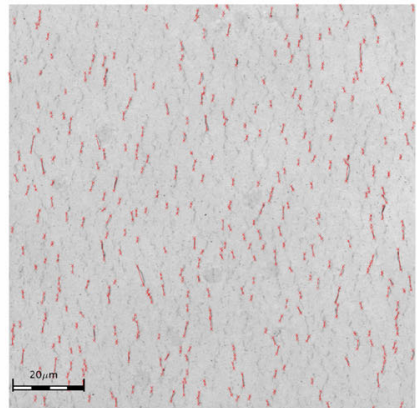

(h) cycle $22, \gamma=6.5$

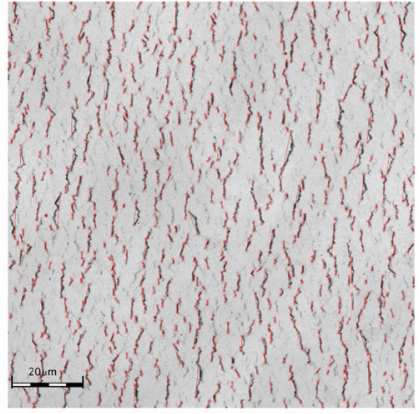

(k) cycle $148, \gamma=6.5$

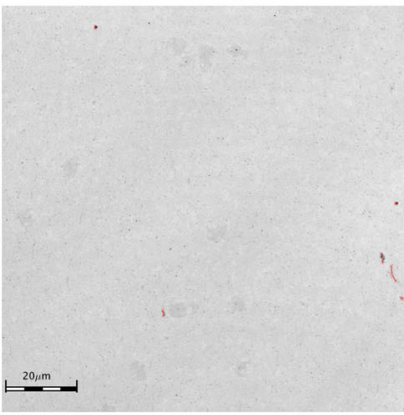

(c) cycle $1, \gamma=8.0$

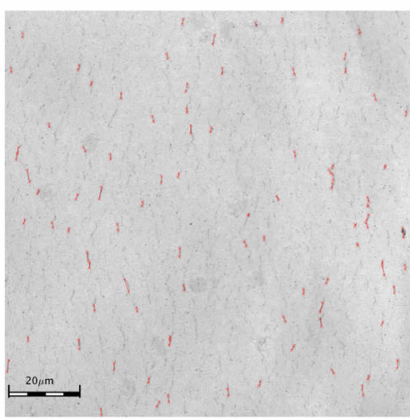

(f) cycle $11, \gamma=8.0$

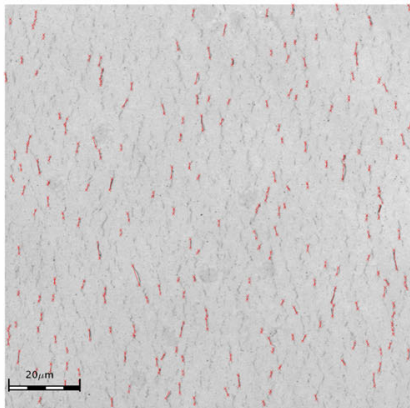

(i) cycle $22, \gamma=8.0$

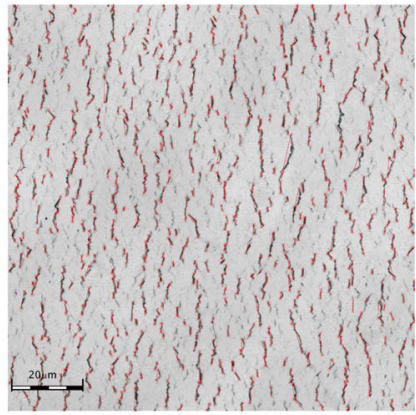

(l) cycle $148, \gamma=8.0$

Fig. 4 Comparison of three different filter options. Depicted are $114 \mu \mathrm{m} \times 114 \mu \mathrm{m}$ micrographs for $\gamma \in\{4.0,6.5,8.0\}$ with red lines indicating the cracks that have been identified 
Table 2 Number of cracks that have been identified for different deformation states by using three different filter options and $114 \mu \mathrm{m} \times 114 \mu \mathrm{m}$ micrographs

\begin{tabular}{lrrrrr}
\hline Filter parameter & \multicolumn{5}{c}{ Cycle } \\
\cline { 2 - 6 } & 1 & 11 & 22 & 123 & 148 \\
\hline$\gamma=4.0$ & 174 & 526 & 764 & 786 & 691 \\
$\gamma=6.5$ & 15 & 153 & 353 & 668 & 598 \\
$\gamma=8.0$ & 7 & 89 & 209 & 588 & 563 \\
\hline
\end{tabular}

ferred formation of cracks orthogonal to the loading direction such that the electric current in $\boldsymbol{e}_{2}$-direction is almost not affected. In addition, small off-diagonal coefficients in the conductivity tensor are induced by the crack formation.

Focussing on the influence of the filter parameter $\gamma$, the projections of $\mathbf{E}_{\mathrm{M}}$ and of $\mathcal{S}_{\mathrm{M}}$ in $\boldsymbol{e}_{1}$-direction for all deformation states and all values of the filter parameter are provided in Fig. 5, and those in $\boldsymbol{e}_{2}$-direction are provided in Fig. 6. In accordance with the presentation of the experimental results in Fig. If and taking into account that

$$
\frac{R}{R_{0}}=\frac{\kappa_{0}}{\kappa} \frac{L}{L_{0}} \frac{A_{0}}{A} \approx \frac{\mathcal{S}_{\mathrm{M} 11}^{\mathrm{ref}}}{\mathcal{S}_{\mathrm{M} 11}^{\bullet}} \frac{L}{L_{0}} \frac{A_{0}}{A}
$$

holds, the simulation results are normalised with respect to an idealised material which does not contain cracks at the microscale. Focusing on the 11-coefficient of the conductivity tensor presented in Fig. 5a in terms of its normalised inverse $\mathcal{S}_{\mathrm{M} 11}^{\mathrm{ref}} / \mathcal{S}_{\mathrm{M} 11}^{\bullet}$ that can be interpreted in terms of the electrical resistivity ratio, a significant increase in $\mathcal{S}_{\mathrm{M} 11}^{\text {ref }} / \mathcal{S}_{\mathrm{M} 11}^{\bullet}$ with increasing deformation and an overall exponential behaviour is observable, which is in good agreement with the experimental findings presented in Fig. 1f. Regarding the influence of the filter parameter $\gamma$, a significant increase in $\mathcal{S}_{\mathrm{M} 11}^{\text {ref }} / \mathcal{S}_{\mathrm{M} 11}^{\bullet}$ with decreasing values of $\gamma$ is found since the number of regions that are identified with cracks increases. In addition, the load-conductivity curve deviates from the experimentally observed exponential-type response for small values of $\gamma$, e.g. for $\gamma=4.0$. Analogous to the electrical conductivity, an exponential decrease is observable in the 1111-coefficient of the stiffness tensor with increasing deformation that becomes more pronounced with decreasing values of $\gamma$. The reference value for the mechanical stiffness tensor $\mathbf{E}_{M}^{\text {ref }}$ used in Fig. 5b is defined by (13) and represents an ide- alised material without cracks. In contrast to the 11coefficient of the conductivity tensor, there is no significant influence of the cracks on $\mathcal{S}_{\mathbf{M} 22}^{\bullet}$, see Fig. 6 a. However, a decrease of approximately $20 \%$ in the 2222 coefficient of the stiffness tensor is found in Fig. $6 \mathrm{~b}$. The different influence of the cracks on the electrical and mechanical properties can be explained by their effect on the lateral contraction, since $\mathrm{E}_{\mathrm{M} 2222}$ measures changes in $\sigma_{\mathrm{M}} 22$ when perturbing $\varepsilon_{\mathrm{M}} 22$ while keeping all other coefficients of $\varepsilon_{\mathrm{M}}$ fixed.

\subsection{Comparison of different micrograph sizes}

Effective macroscopic material properties are calculated in multiscale finite element simulations based on detailed information on the underlying microstructure and crack pattern. Against this background, the micrographs must be representative for a particular material region. To ensure that the respective micrographs contain sufficient information, the convergence behaviour of the effective macroscopic material properties on micrograph-size-enlargement is studied in this section. Being more specific, $38 \mu \mathrm{m} \times 38 \mu \mathrm{m}, 76 \mu \mathrm{m} \times 76 \mu \mathrm{m}$ and $114 \mu \mathrm{m} \times 114 \mu \mathrm{m}$ micrographs, measured relative to the upper left corner, are prepared from the full-sized micrographs depicted in Fig. 1. To allow for a good comparability of the results, all of the micrographs are processed with the same filter parameters, i.e. $\gamma=6.5$ is used and geometric objects that have fewer than 15 pixels are removed. The discrete representations of the microstructures that have been created by using this procedure are provided in Fig. 7 and in Fig. 8.

Focussing exemplarily on the conductivity tensors that were calculated for cycle 123 and for the three different micrograph sizes, cf. Fig. 7 ,

$$
\begin{aligned}
{\left[\mathcal{S}_{\mathrm{M}}\right]_{i j}^{38 \mu \mathrm{m}} } & =\left[\begin{array}{rr}
0.8447 & -0.0079 \\
-0.0079 & 0.9913
\end{array}\right] \kappa \\
{\left[\mathcal{S}_{\mathrm{M}}\right]_{i j}^{76 \mu \mathrm{m}} } & =\left[\begin{array}{rr}
0.8303 & 0.0034 \\
0.0034 & 0.9905
\end{array}\right] \kappa \\
{\left[\mathcal{S}_{\mathrm{M}}\right]_{i j}^{114 \mu \mathrm{m}} } & =\left[\begin{array}{ll}
0.8173 & 0.0015 \\
0.0015 & 0.9912
\end{array}\right] \kappa
\end{aligned}
$$

only a small difference in the 11-coefficient of approximately $0.0144 \kappa(1.70 \%)$ between the $38 \mu \mathrm{m} \times 38 \mu \mathrm{m}$ and the $76 \mu \mathrm{m} \times 76 \mu \mathrm{m}$ micrograph, and of approximately $0.0130 \kappa(1.56 \%)$ between the $76 \mu \mathrm{m} \times 76 \mu \mathrm{m}$ 


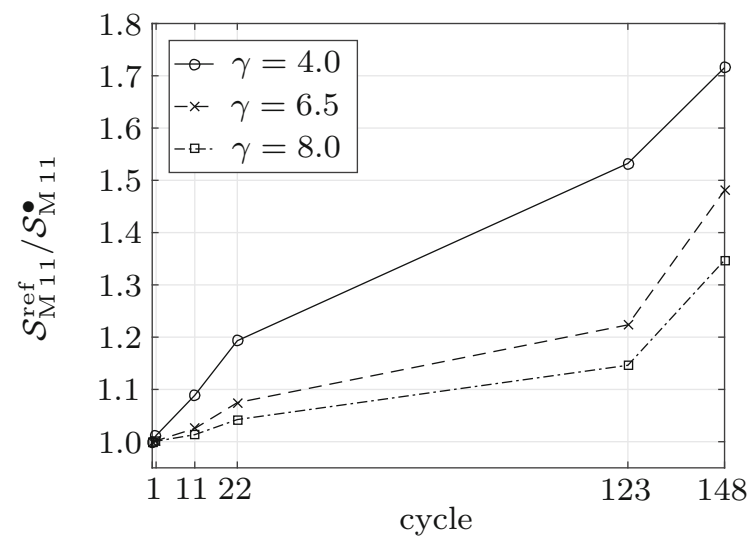

(a) normalised conductivity

Fig. 5 Comparison of simulation results in terms of normalised conductivity and stiffness coefficients that result from the projection of $\mathcal{S}_{M}$ and of $\mathbf{E}_{\mathrm{M}}$ in $\boldsymbol{e}_{1}$-direction. The respective coefficients

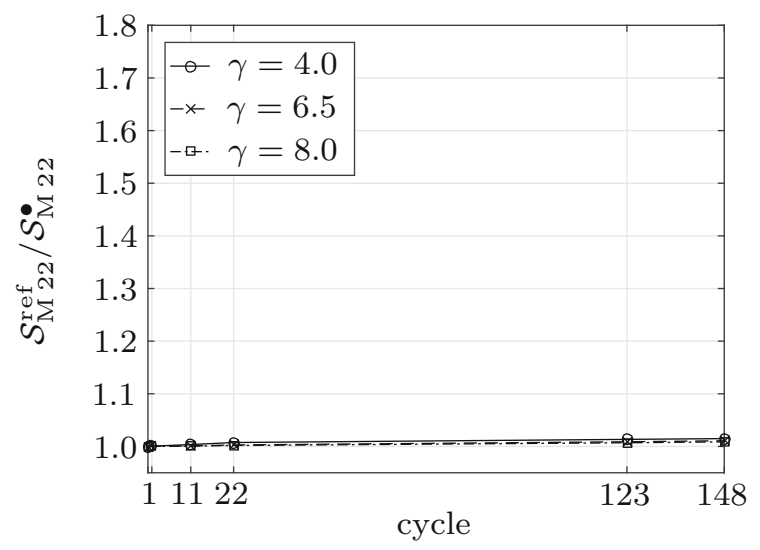

(a) normalised conductivity

Fig. 6 Comparison of simulation results in terms of normalised conductivity and stiffness coefficients that result from the projection of $\mathcal{S}_{\mathrm{M}}$ and of $\mathbf{E}_{\mathrm{M}}$ in $\boldsymbol{e}_{2}$-direction. The respective coefficients

and the $114 \mu \mathrm{m} \times 114 \mu \mathrm{m}$ micrograph is observed. In addition, the projections of the normalised conductivity and of the normalised stiffness tensors in $\boldsymbol{e}_{1}$-direction are provided in Fig. 9, and those in $\boldsymbol{e}_{2}$-direction are provided in Fig. 10 for all deformation states. The coefficients of the conductivity tensor $\mathcal{S}_{\mathrm{M} 11}^{\bullet}$ are provided in Table 3 in a normalised form for all deformation states and micrographs of different size. Overall, and in particular when compared with the influence of different filter options, the influence of the micrograph size on the simulation results is small.

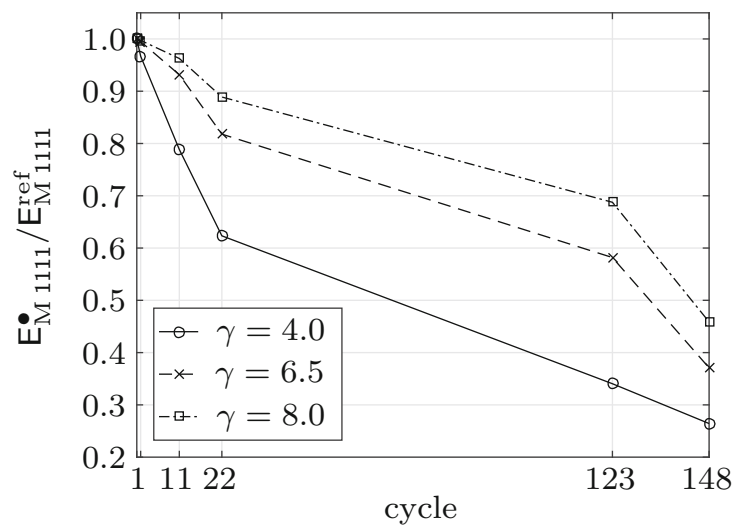

(b) normalised stiffness

are presented for different filter options and load states according to Figs. 3 and 4

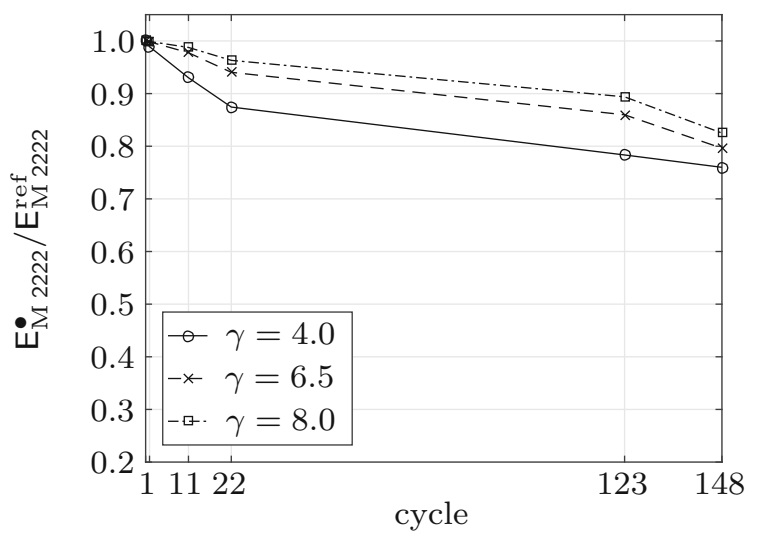

(b) normalised stiffness

are presented for different filter options and load states according to Figs. 3 and 4

\subsection{Comparison of different mesh sizes}

This section focuses on the influence of the finite element discretisation on the macroscopic material properties that are calculated by using the computational homogenisation scheme proposed in Sect. 3.2. To this end, three different discretisations of the $114 \mu \mathrm{m} \times$ $114 \mu \mathrm{m}$ micrographs are analysed which are processed with the same filter options, i.e. $\gamma=6.5$ is used and geometric objects that have fewer than 15 pixels are removed. From a numerical point of view, quadratic 


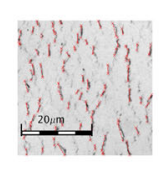

(a) $38 \mu \mathrm{m} \times 38 \mu \mathrm{m}$

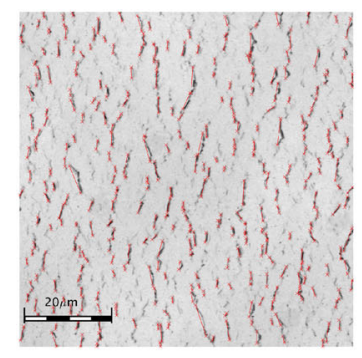

(b) $76 \mu \mathrm{m} \times 76 \mu \mathrm{m}$

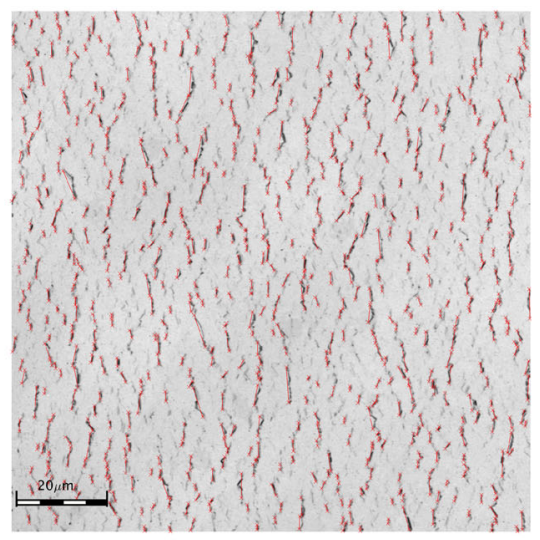

(c) $114 \mu \mathrm{m} \times 114 \mu \mathrm{m}$

Fig. 7 Comparison of different micrograph sizes. Depicted are $38 \mu \mathrm{m} \times 38 \mu \mathrm{m}, 76 \mu \mathrm{m} \times 76 \mu \mathrm{m}$ and $114 \mu \mathrm{m} \times 114 \mu \mathrm{m}$ micrographs of cycle 123 with $\gamma=6.5$ and with red lines indicating cracks that haven been identified

triangular elements are used for the discretisation of the geometry, of the displacement field $\boldsymbol{u}$ and of the electric potential field $\phi$. Integrals are evaluated numerically by using a quadrature scheme with three integration points. The resulting numbers of elements of the coarse, medium and fine discretisations for all deformation states are summarised in Table 4. Moreover, it is noted that the results presented in Sect. 4.1 and in Sect. 4.2 are based on discretisations with a medium mesh size.

The coefficient matrices of the electrical conductivity tensors for cycle 123 and the $114 \mu \mathrm{m} \times 114 \mu \mathrm{m}$ micrographs that were processed with $\gamma=6.5$ are given by

$$
\begin{aligned}
{\left[\mathcal{S}_{\mathrm{M}}\right]_{i j}^{\text {coarse }} } & =\left[\begin{array}{ll}
0.8266 & 0.0013 \\
0.0013 & 0.9917
\end{array}\right] \kappa \\
{\left[\mathcal{S}_{\mathrm{M}}\right]_{i j}^{\text {medium }} } & =\left[\begin{array}{ll}
0.8173 & 0.0015 \\
0.0015 & 0.9912
\end{array}\right] \kappa \\
{\left[\mathcal{S}_{\mathrm{M}}\right]_{i j}^{\mathrm{fine}} } & =\left[\begin{array}{ll}
0.8099 & 0.0016 \\
0.0016 & 0.9908
\end{array}\right] \kappa
\end{aligned}
$$

The differences in the 11-coefficient between the coarse and medium discretisation of approximately $0.0093 \kappa$ $(1.13 \%)$ as well as the one between the medium and the fine discretisation of approximately $0.0074 \kappa(0.91 \%)$ are found to be small. This observation is further underlined by the projections in 11-direction of the normalised conductivity and of the normalised stiffness tensors presented in Fig. 11. In addition to the graphical representation, the normalised 11-coefficients of the conductivity tensors are provided in Table 5 for the different discretisations and deformation states.

\section{Comparison of simulation results with experimental data}

This section concerns the comparison of the experimentally recorded relative resistance ratio as a function of mechanical damage with multiscale simulation results. Since the cracks that are geometrically resolved for each individual load step, and hence also the corresponding representative volume elements, depend on the filter parameter $\gamma$ as has been shown in Sect. 4.1, the laser intensity images are again subjected to a detailed analysis. Therefore, the number of cracks crossing certain cutting planes will be taken into account to assess the quality of the representative volume elements for different filter values. For load cycle 123 and $\gamma=6.5$, the cutting planes are exemplarily indicated by bluecoloured (horizontal) lines in Fig. 12a. Since the number of cracks crossing the cutting planes in the filtered CLSM images is sensitive with regard to slight perturbations of the line position, 12 cutting planes are taken into account for each deformation state and each filter value-i.e. four primary cutting planes, indicated by solid blue-coloured lines, and another 8 secondary cutting planes at $\pm 0.64 \mu \mathrm{m}$, indicated by dashed blue- 


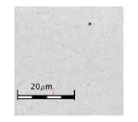

(a) cycle 1

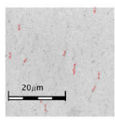

(d) cycle 11

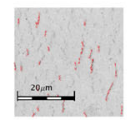

(g) cycle 22

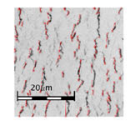

(j) cycle 148

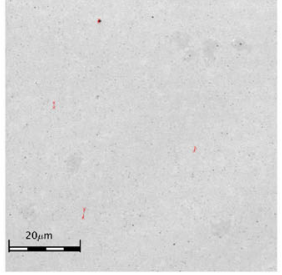

(b) cycle 1

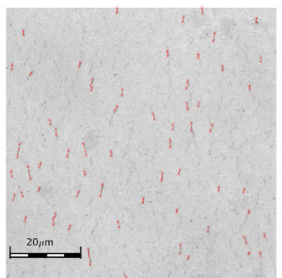

(e) cycle 11

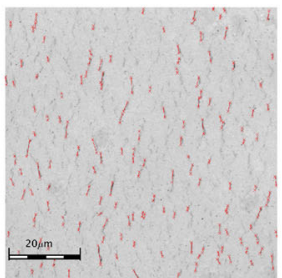

(h) cycle 22

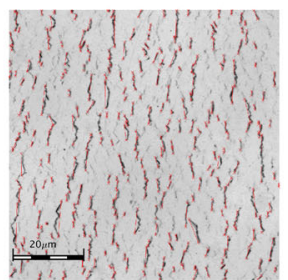

(k) cycle 148

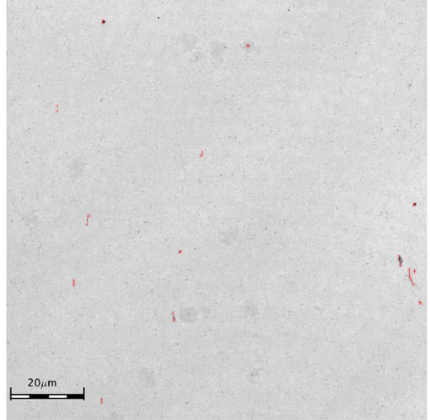

(c) cycle 1

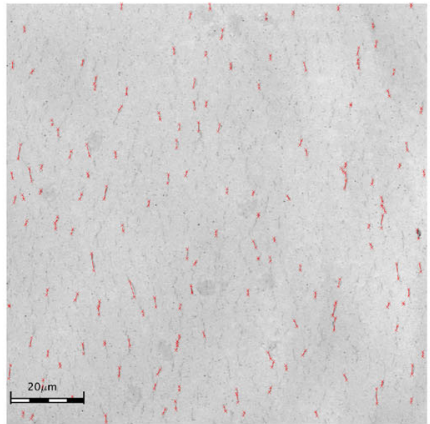

(f) cycle 11

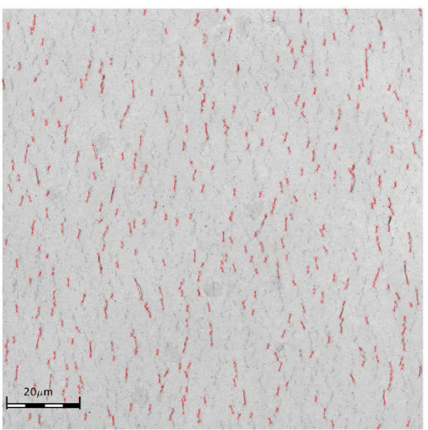

(i) cycle 22

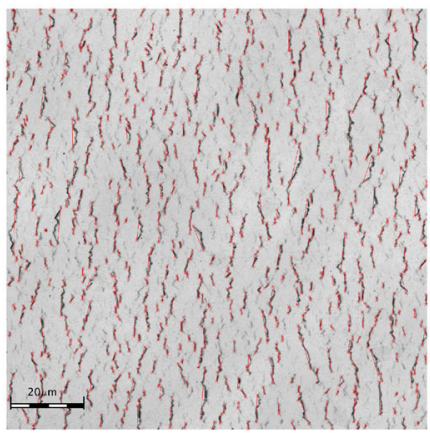

(I) cycle 148

Fig. 8 Comparison of different micrograph sizes. Depicted are $38 \mu \mathrm{m} \times 38 \mu \mathrm{m}, 76 \mu \mathrm{m} \times 76 \mu \mathrm{m}$ and $114 \mu \mathrm{m} \times 114 \mu \mathrm{m}$ micrographs of different deformation states with $\gamma=6.5$ and with red lines indicating cracks that have been identified 


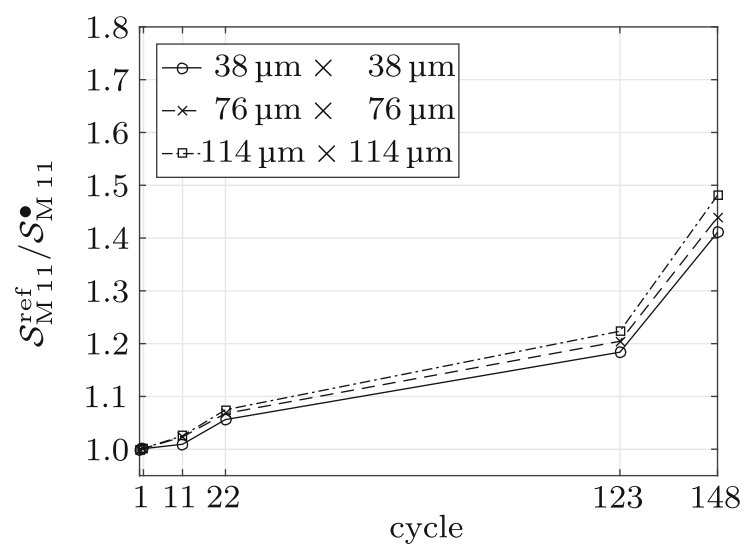

(a) normalised conductivity

Fig. 9 Comparison of simulation results in terms of normalised conductivity and stiffness coefficients that result from the projection of $\mathcal{S}_{\mathrm{M}}$ and of $\mathbf{E}_{\mathrm{M}}$ in $\boldsymbol{e}_{1}$-direction. The respective coefficients

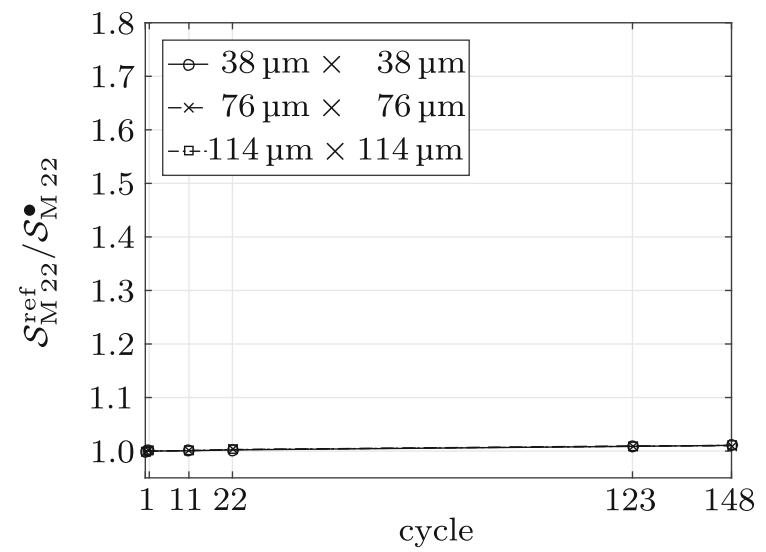

(a) normalised conductivity

Fig. 10 Comparison of simulation results in terms of normalised conductivity and stiffness coefficients that result from the projection of $\mathcal{S}_{\mathrm{M}}$ and of $\mathbf{E}_{\mathrm{M}}$ in $\boldsymbol{e}_{2}$-direction. The respective coefficients

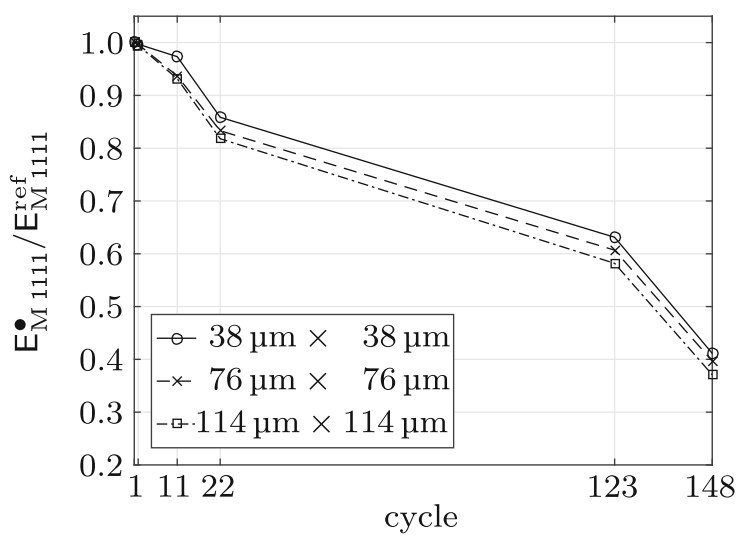

(b) normalised stiffness

are presented for $\gamma=6.5$, for different micrograph sizes and for load states according to Figs. 7 and 8

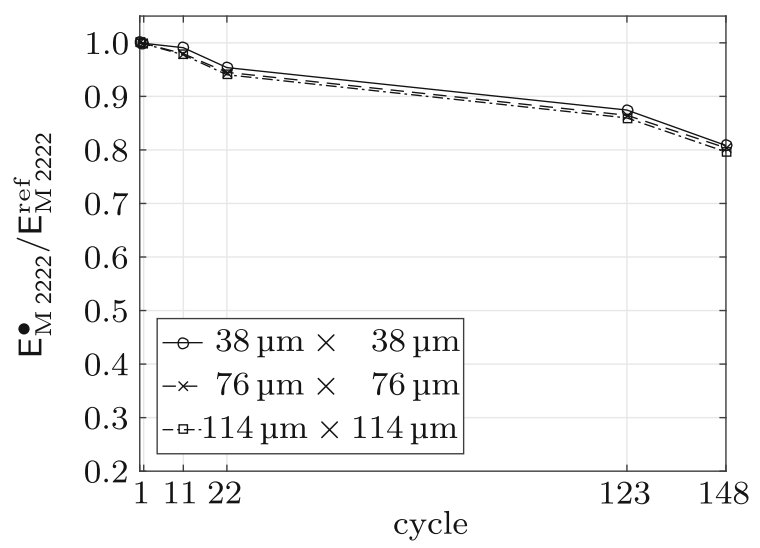

(b) normalised stiffness

are presented for $\gamma=6.5$, for different micrograph sizes and for load states according to Figs. 7 and 8

Table 3 Coefficients of the normalised conductivity tensor $\mathcal{S}_{\mathrm{M} 11}^{\text {ref }} / \mathcal{S}_{\mathrm{M} 11}^{\bullet}$ for different micrograph sizes, different deformation states and $\gamma=6.5$

\begin{tabular}{lllll}
\hline Micrograph & \multicolumn{4}{c}{ Cycle } \\
\cline { 2 - 5 } & 1 & 11 & 22 & 123 \\
\hline $38 \mu \mathrm{m}$ & 1.0011 & 1.0094 & 1.0562 & 1.1838 \\
$76 \mu \mathrm{m}$ & 1.0012 & 1.0234 & 1.0677 & 1.2044 \\
$114 \mu \mathrm{m}$ & 1.0017 & 1.0253 & 1.0747 & 1.2235 \\
\hline
\end{tabular}


Table 4 Number of elements used in the three different discretisations of the $114 \mu \mathrm{m} \times 114 \mu \mathrm{m}$ micrographs that are processed with $\gamma=6.5$

\begin{tabular}{|c|c|c|c|c|c|}
\hline \multirow[t]{2}{*}{ Mesh size } & \multicolumn{5}{|c|}{ Cycle } \\
\hline & 1 & 11 & 22 & 123 & 148 \\
\hline Coarse & 24222 & 47260 & 42104 & 38492 & 28084 \\
\hline Medium & 44194 & 64750 & 71212 & 75724 & 72054 \\
\hline Fine & 81048 & 191092 & 238466 & 253292 & 258760 \\
\hline
\end{tabular}

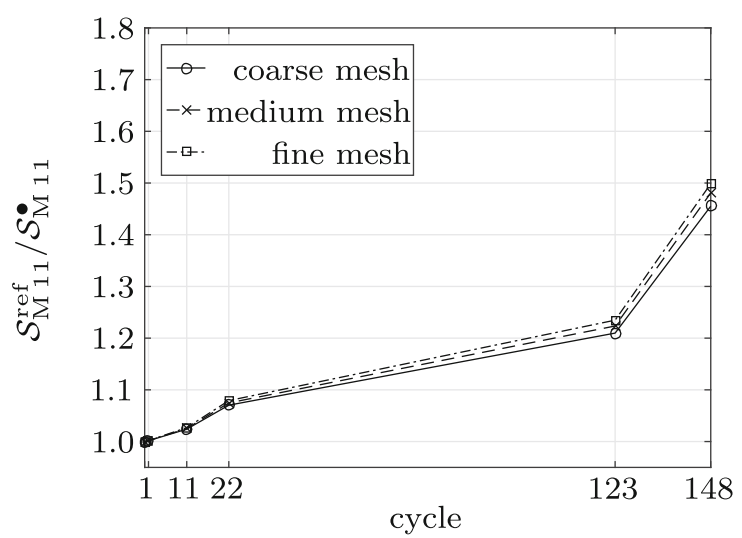

(a) normalised conductivity

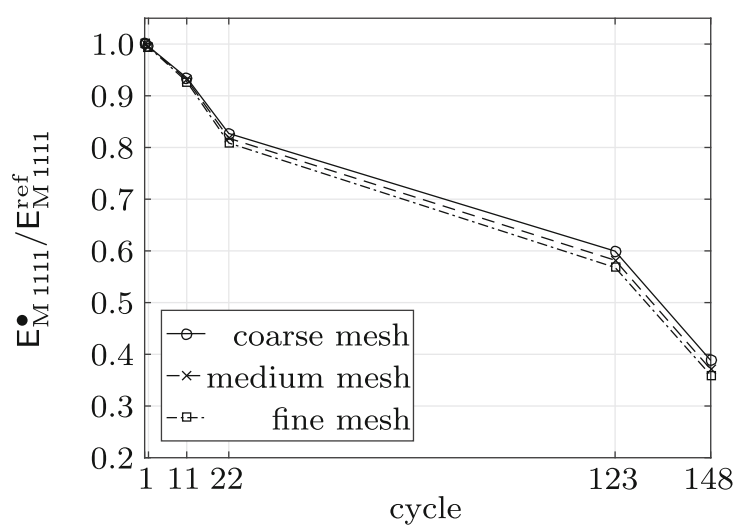

(b) normalised stiffness

Fig. 11 Comparison of simulation results in terms of normalised conductivity and normalised stiffness coefficients for different finite element meshes according to Table 4. The calculations are based on $\gamma=6.5$ and on $114 \mu \mathrm{m} \times 114 \mu \mathrm{m}$ micrographs

Table 5 Coefficients of the normalised electrical conductivity tensor $\mathcal{S}_{\mathrm{M} 11}^{\text {ref }} / \mathcal{S}_{\mathrm{M} 11}^{\bullet}$ for $114 \mu \mathrm{m} \times 114 \mu \mathrm{m}$ micrographs, different mesh sizes, different deformation states and $\gamma=6.5$

\begin{tabular}{llllll}
\hline Mesh size & \multicolumn{3}{c}{ Cycle } & 123 & 148 \\
\cline { 2 - 6 } & 1 & 11 & 22 & 1.2098 & 1.4552 \\
Coarse & 1.0016 & 1.0240 & 1.0704 & 1.2235 & 1.4808 \\
Medium & 1.0017 & 1.0253 & 1.0747 & 1.2348 & 1.4985 \\
Fine & 1.0019 & 1.0269 & 1.0792 & \\
\hline
\end{tabular}

coloured lines. The respective mean values are provided in Table 6 and are compared against the number of cracks that stem from a detailed analysis of the CLSM laser intensity profiles by hand corresponding to the primary cutting planes. For cycle 123 and cutting plane A the CLSM laser intensity profile is shown in Fig. 12b. TTCs manifest themselves as steep gradients (drops in intensity) in CLSM laser intensity images. In this regard, the dashed red-coloured line in Fig. 12b indicates the chosen tolerance-i.e. (local) minima in the laser intensity profile that are smaller than the tolerance, chosen for this particular CLSM image and for this particular cutting plane, are counted as TTCs when the state of the art evaluation method that relies on a manual evaluation of the crack-density is applied. Minima that take higher values are associated with necks. This procedure is in line with the calculation of the linear crack density as described in Cordil et al. (2017) and Glushko et al. (2020). It is noted that the definition of the crack density is not unique and that the crack density may be introduced differently as briefly outlined in Remark 2.

A comparison of the values in Table 6 demonstrates the applicability of the automated image analysis meth- 


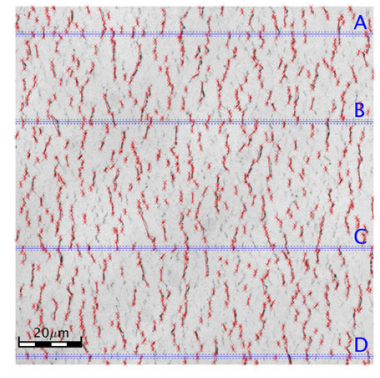

(a) filtered CLSM laser intensity image

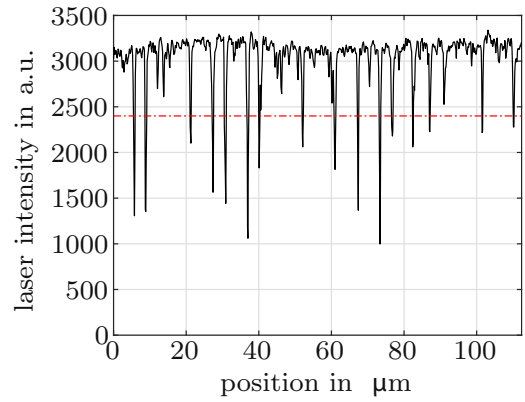

(b) CLSM laser intensity profile

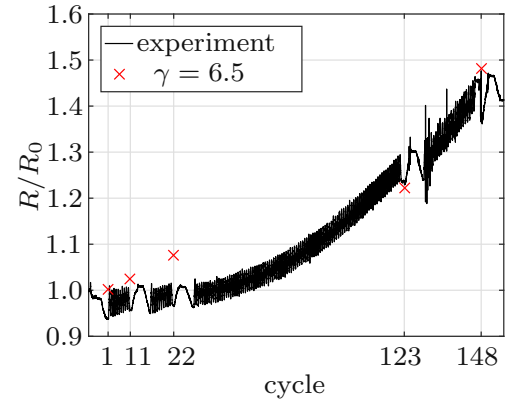

(c) relative resistance ratio
Fig. 12 Exemplary evaluation of damage density based on a $114 \mu \mathrm{m} \times 114 \mu \mathrm{m}$ micrograph representing cycle 123 , and comparison of experiments with simulation results. a CLSM intensity image that was processed by using a filter value $\gamma=6.5$. Red lines indicate cracks that have been identified. Blue lines indicate cross sections for which the damage density is evaluated. $\mathbf{b}$
CLSM laser intensity profile along cross-section A for cycle 123. The red line indicates the chosen tolerance for TCCs. c Experimentally recorded relative resistance ratio as a function of load cycles, and multiscale simulation results for $114 \mu \mathrm{m} \times 114 \mu \mathrm{m}$ micrographs, $\gamma=6.5$ and a medium mesh size ods used in the RVE generation process. For each step the values determined manually correspond well to the filter parameters of 6.5 and 8.0-certainly, there is some arbitrariness in the choice of the filter parameter, with the latter comparison establishing mathematical bounds for reasonable values of $\gamma$. Image analysis methods, such as the one applied here, do not only allow for certain cutting planes, but for the entire image to be evaluated. Up until now it has not been possible to assess every crack in an image. Analysing the entire image provides more and better statistics on the mechanical damage (TTC) and its evolution with increasing cycle number.

Based on the comparison of the mean number of cracks crossing the cutting planes, the experimentally recorded relative resistance ratio is compared with simulation results for $\gamma=6.5$ in a first step. The experimental data is provided in Fig. 12c along with the relative resistance ratio for cycles $1,11,22,123$, and 148 , calculated by means of multiscale finite element simulations. In general, and especially for high cycle numbers, experiment and simulation are in good agreement. However, there is an interesting difference at cycle 22 which suggests that for small cycle numbers too many regions have been associated with TTCs which leads to an increase in the predicted relative resistance ratio. Comparing the corresponding micrograph of cycle 22 shown in Fig. 1c with the images corresponding to higher cycle numbers, e.g. Fig. 1d or e, it is observed that the greyscale gradients are much less pronounced.
Accordingly, this observation underlines the need for further experimental and simulation based analyses in future works, in order to reliably distinguish between TTCs and necks. In this regard, the development of more elaborated, tailored filters is considered to be key. Nevertheless, the comparison of simulation results and experimental findings clearly demonstrates the usefulnesses of the proposed multiscale formulation in relating microscale cracks to macroscopic electrical properties of metal thin films.

Remark 2 Different definitions of the crack density as a characteristic of the microstructure may be introduced. It is for instance proposed in Kanaun and Levin (2008) to work with crack densities $\tau$ of the type

$\tau=\mathrm{n}_{\mathrm{cra}}\left\langle\pi l^{2}\right\rangle$

where $\mathrm{n}_{\text {cra }}$ denotes the number of cracks per unit area, $l$ is half the crack length and $\langle\bullet\rangle$ indicates the mean value with regard to the crack set.

In this regard, it is noted that parameters $\tau$ and $l$ in (22) are intrinsically filter-dependent such that unique values of these parameters may not be provided for the experimental images in Fig. 1, see also the dicussion in Sect. 5. 
Table 6 Mean number of cracks per cross section calculated for different filter parameters based on the CLSM laser intensity images. The mean number of cracks per cross section is calculated based on the $114 \mu \mathrm{m} \times 114 \mu \mathrm{m}$ micrographs

\begin{tabular}{llllll}
\hline Filter parameter & \multicolumn{4}{c}{ Cycle } & \\
\cline { 2 - 5 } & 1 & 11 & 22 & 123 & 148 \\
\hline$\gamma=4.0$ & 1.25 & 6.41 & 12.75 & 17.58 & 17.25 \\
$\gamma=6.5$ & 0.33 & 1.50 & 5.25 & 12.00 & 13.75 \\
$\gamma=8.0$ & 0.16 & 0.83 & 3.00 & 9.58 & 12.00 \\
CLSM (manually) & 0.00 & 2.00 & 4.50 & 8.50 & 13.25 \\
\hline
\end{tabular}

\section{Closure}

In this contribution, the influence of the crack pattern at the microscale on effective electrical and mechanical properties at the macroscale was studied. To this end, macroscopic conductivity and stiffness tensors were calculated based on experimentally obtained micrographs and compared against experimental results.

In a first step, representative volume elements with geometrically resolved cracks were generated from confocal laser scanning microscopy images by using image processing techniques. Next, a computational homogenisation approach was followed to extract effective macroscopic conductivity and stiffness tensors from the representative volume elements. It was then shown that the filter used in the image processing step in order to identify cracks has a significant influence on the predicted macroscopic properties. Moreover, convergence studies revealed only a small influence of the representative volume element size and of the finite element discretisation on the simulation results. The comparison of simulation results with experimentally recorded load-resistance curves eventually demonstrated the usefulness of the proposed multiscale formulation for studying the influence of microscale cracks on the effective electrical material properties of metal thin films.

From an experimental point of view, future work will focus on further detailed analyses of micrographs containing cracks by using, e.g. 3D height images (AFM or CLSM) from uniaxial monotonic or cyclic straining as well as cyclic bending experiments, in order to elucidate which areas in the images are cracks and which areas are plastic localisation zones. This will contribute to the calibration and to the development of tailored image processing techniques for crack identi- fication. From a simulation point of view, experimentbased material models and formulations that allow for predictive simulations of the crack formation on the microscale need to be developed. Together with the computational homogenisation scheme presented in this contribution, these will eventually allow for the simulation of changes in effective macroscopic material properties based on spatially resolved microstructure and crack pattern evolutions.

Acknowledgements Open Access funding enabled and organized by Projekt DEAL. Financial support by the Deutsche Forschungsgemeinschaft (DFG, German Research Foundation) Project-ID 278868966-TRR 188 is gratefully acknowledged.

Funding Open Access funding enabled and organized by Projekt DEAL.

\section{Declarations}

Conflict of interest On behalf of all authors, the corresponding author states that there is no conflict of interest.

Open Access This article is licensed under a Creative Commons Attribution 4.0 International License, which permits use, sharing, adaptation, distribution and reproduction in any medium or format, as long as you give appropriate credit to the original author(s) and the source, provide a link to the Creative Commons licence, and indicate if changes were made. The images or other third party material in this article are included in the article's Creative Commons licence, unless indicated otherwise in a credit line to the material. If material is not included in the article's Creative Commons licence and your intended use is not permitted by statutory regulation or exceeds the permitted use, you will need to obtain permission directly from the copyright holder. To view a copy of this licence, visit http://creativecommons.org/licenses/ by/4.0/. 


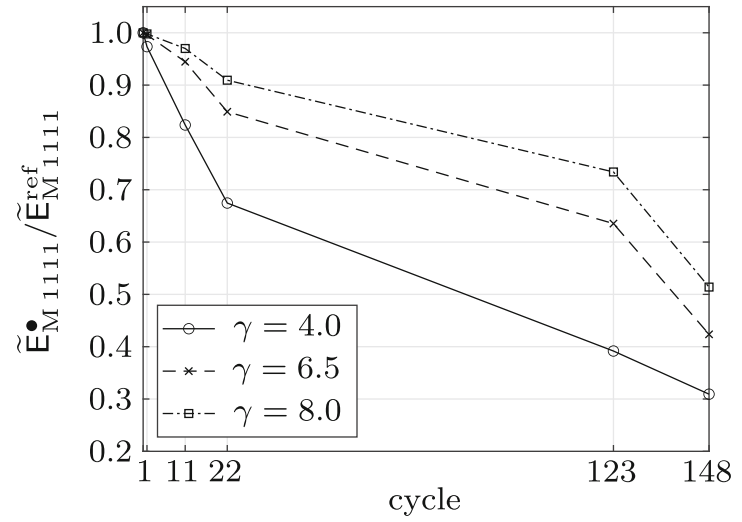

(a) stiffness projection in $\boldsymbol{e}_{1}$-direction

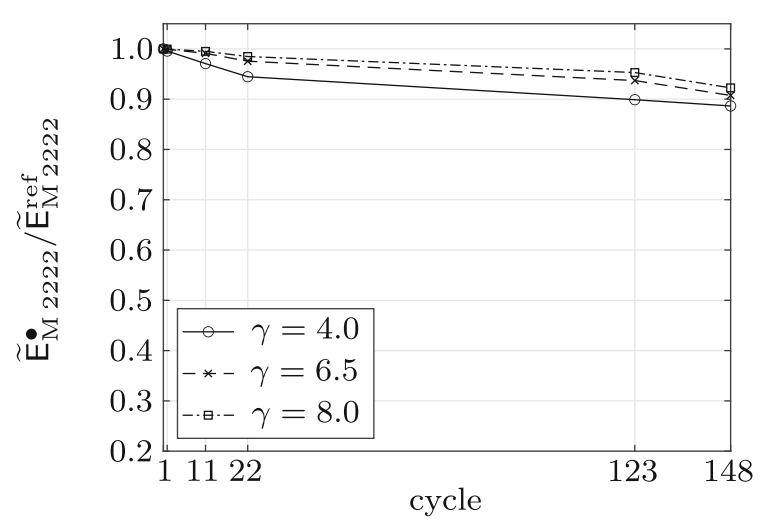

(b) stiffness projection in $\boldsymbol{e}_{2}$-direction

Fig. 13 Projection of $\widetilde{\mathbf{E}}_{\mathrm{M}}$ in $\boldsymbol{e}_{1}$ - and $\boldsymbol{e}_{2}$-direction for different filter options and load states according to Figs. 3 and 4

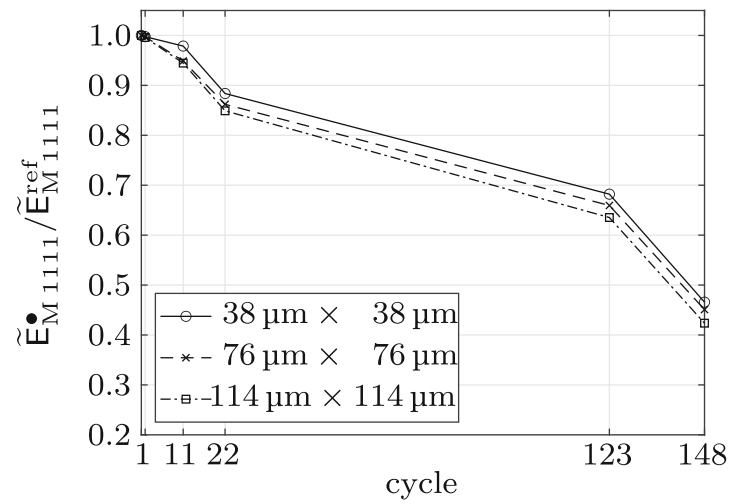

(a) stiffness projection in $\boldsymbol{e}_{1}$-direction

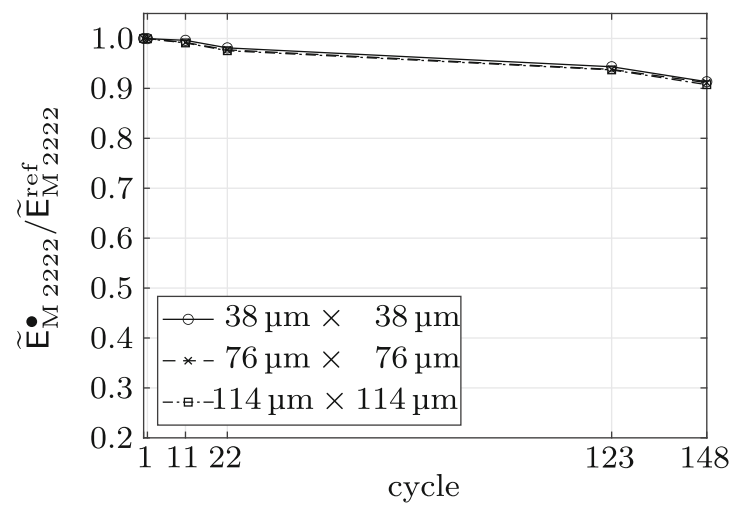

(b) stiffness projection in $e_{2}$-direction

Fig. 14 Projection of $\widetilde{\mathbf{E}}_{\mathrm{M}}$ in $\boldsymbol{e}_{1}$ - and $\boldsymbol{e}_{2}$-direction for $\gamma=6.5$, for different micrograph sizes and for load states according to Figs. 7 and 8

\section{Appendix A: Plane stress deformation states}

For the sake of comparison, simulation results that are based on a plane stress assumption are additionally provided in this appendix. To this end, the fourth-order two-dimensional plane stress elasticity tensor

$$
\widetilde{\mathbf{E}}_{\mathrm{m}}=\tilde{\lambda} \boldsymbol{I} \otimes \boldsymbol{I}+\mu[\boldsymbol{I} \bar{\otimes}+\boldsymbol{I} \underline{\otimes} \boldsymbol{I}]
$$

with

$$
\tilde{\lambda}=\frac{E v}{1-v^{2}}
$$

is introduced. The projections of the corresponding homogenised macroscale tensor $\widetilde{\mathbf{E}}_{\mathrm{M}}$ in $\boldsymbol{e}_{1}$ - and $\boldsymbol{e}_{2}$ direction for different values of the filter parameter $\gamma$ and for different micrograph sizes are provided in Figs. 13 and 14.

\section{References}

Berger J, Glushko O, Marx VM, Kirchlechner C, Cordill MJ (2016) Effect of microstructure on the electro-mechanical behaviour of $\mathrm{Cu}$ films on polyimide. JOM 68:1640-1646. https://doi.org/10.1007/s11837-016-1940-z

Berthelsen R, Menzel A (2019) Computational homogenisation of thermo-viscoplastic composites: large strain formulation and weak micro-periodicity. Comput Methods Appl Mech Eng 348:575-603. https://doi.org/10.1016/j.cma.2018.12. 032

Berthelsen R, Denzer R, Oppermann P, Menzel A (2017) Computational homogenisation for thermoviscoplasticity: application to thermally sprayed coatings. 
Comput Mech 60(5):739-766. https://doi.org/10.1007/ s00466-017-1436-x

Cahn G, Barrios A, Graham S, Meth J, Antoniou A, Pierron O (2020) The role of strain localization on the electrical behavior of flexible and stretchable screen printed silver inks on polymer substrates. Materialia 10:100642. https:// doi.org/10.1016/j.mtla.2020.100642

Coenen EWC, Kouznetsova VG, Bosco E, Geers MGD (2012) A multi-scale approach to bridge microscale damage and macroscale failure: a nested computational homogenization-localization framework. Int J Fract 178:157-178. https://doi.org/10.1007/s10704-012-9765-4

Coenen EWC, Kouznetsova VG, Geers MGD (2012) Multi-scale continuous-discontinuous framework for computationalhomogenization-localization. J Mech Phys Solids 60(8):1486-1507. https://doi.org/10.1088/0965-0393/ 19/7/074008

Cordil MJ, Glushko O, Kleinbichler A, Putz B, Többens DM, Kirchlechner C (2017) Microstructural influence on the cyclic electro-mechanical behaviour of ductile films on polymer substrates. Thin Solid Films 644:166-172. https:// doi.org/10.1016/j.tsf.2017.06.067

Cordill MJ, Glushko O, Kreith J, Marx VM, Kirchlechner C (2015) Measuring electro-mechanical properties of thin films on polymer substrates. Microelectron Eng 137:96100. https://doi.org/10.1016/j.mee.2014.08.002

Cordill MJ, Glushko O, Putz B (2016) Electro-mechanical testing of conductive materials used in flexible electronics. Front Mater 3:1-11. https://doi.org/10.3389/fmats.2016.00011

Cordill MJ, Marx VM (2013) Fragmentation testing for ductile thin films on polymer substrates. Philos Mag Lett 93(11):618-624. https://doi.org/10.1080/09500839.2013. 830792

Cordill MJ, Marx VM (2013) In-situ tensile straining of metal films on polymer substrates under an AFM. MRS Proc. https://doi.org/10.1557/opl.2013.617

Cordill MJ, Taylor A, Schalko J, Dehm G (2010) Fracture and delamination of chromium thin films on polymer substrates. Metall Mater Trans A 41(4):870-875. https://doi.org/10. 1007/s11661-009-9988-9

Etiemble A, Lopes C, Ingrid GNB, Borges J, Malchère A, Langlois C, Vaz F, Steyer P (2019) Fracture resistance of Ti-Ag thin films deposited on polymeric substrates for biosignal acquisition applications. Surf Coat Technol 358:646-653. https://doi.org/10.1016/j.surfcoat.2018.11.078

Feyel F, Chaboche JL (2000) $\mathrm{FE}^{2}$ multiscale approach for modelling the elastoviscoplastic behaviour of long fibre $\mathrm{SiC} / \mathrm{Ti}$ composite materials. Comput Methods Appl Mech Eng 183(3):309-330. https://doi.org/10.1016/ S0045-7825(99)00224-8

Glushko O, Cordill MJ (2016) Electrical resistance of metal films on polymer substrates under tension. Exp Tech 40:303-310. https://doi.org/10.1111/ext.12082

Glushko O, Klug A, List-Kratochvil EJ, Cordill MJ (2017) Monotonic and cyclic mechanical reliability of metallization lines on polymer substrates. J Mater Res 32(9):17601769. https://doi.org/10.1557/jmr.2017.121

Glushko O, Putz B, Cordill MJ (2020) Determining effective crack lengths from electrical measurements in polymersupported thin films. Thin Solid Films 699:137906. https:// doi.org/10.1016/j.tsf.2020.137906
Gruber PA, Arzt E, Spolenak R (2009) Brittle-to-ductile transition in ultrathin $\mathrm{Ta} / \mathrm{Cu}$ film systems. J Mater Res 24(6):1906-1918. https://doi.org/10.1557/jmr.2009.0252

Gu T, Castelnau O, Forest S, Hervé-Luanco E, Lecouturier F, Proudhon H, Thilly L (2017) Multiscale modeling of the elastic behavior of architectured and nanostructured $\mathrm{Cu}-\mathrm{Nb}$ composite wires. Int J Solids Struct 121:148-162. https:// doi.org/10.1016/j.ijsolstr.2017.05.022

Jin H, Lu WY, Cordill MJ, Schmidegg K (2011) In situ study of cracking and buckling of chromium films on PET substrates. Exp Mech 51(2):219-227. https://doi.org/10.1007/ s11340-010-9359-x

Kaiser T, Menzel A (2021) An electro-mechanically coupled computational multiscale formulation for electrical conductors. Arch Appl Mech 91:1509-1526. https://doi.org/10. 1007/s00419-020-01837-6

Kaiser T, Menzel A (2021) A finite deformation electromechanically coupled computational multiscale formulation for electrical conductors. Acta Mech. https://doi.org/ 10.1007/s00707-021-03005-5

Kanaun SK, Levin V (2008) Self-consistent methods for composites static problems, vol 148. Springer, Dordrecht

Keip MA, Steinmann P, Schröder J (2014) Two-scale computational homogenization of electro-elasticity at finite strains. Comput Methods Appl Mech Eng 278:62-79. https://doi. org/10.1016/j.cma.2014.04.020

Khalaquzzaman M, Xu BX, Ricker S, Müller R (2012) Computational homogenization of piezoelectric materials using FE2 to determine configurational forces. Tech Mech 32(1):2137

Kouznetsova VG, Brekelmans WAM, Baaijens FPT (2001) An approach to micro-macro modeling of heterogeneous materials. Comput Mech 27(1):37-48. https://doi.org/10.1007/ s004660000212

Kouznetsova VG, Geers MGD, Brekelmans WAM (2002) Multiscale constitutive modelling of heterogeneous materials with a gradient-enhanced computational homogenization scheme. Int J Numer Methods Eng 54(8):1235-1260. https://doi.org/10.1002/nme.541

Kouznetsova VG, Geers MGD, Brekelmans WAM (2004) Multiscale second-order computational homogenization of multiphase materials: a nested finite element solution strategy. Comput Methods Appl Mech Eng 193(48):5525-5550. https://doi.org/10.1016/j.cma.2003.12.073

Kreiml P, Rausch M, Terziyska VL, Köstenbauer H, Winkler J, Mitterer C, Cordill MJ (2019) Correlation of mechanical damage and electrical behavior of Al/Mo bilayers subjected to bending. Thin Solid Films 687:137480. https://doi.org/ 10.1016/j.tsf.2019.137480

Lambricht N, Pardoen T, Yunus S (2013) Giant stretchability of thin gold films on rough elastomeric substrates. Acta Mater 61(2):540-547. https://doi.org/10.1016/j.actamat.2012.10. 001

Leterrier Y, Médico L, Demarco F, Månson JA, Betz U, Escolà M, Kharrazi Olsson M, Atamny F (2004) Mechanical integrity of transparent conductive oxide films for flexible polymerbased displays. Thin Solid Films 460(1-2):156-166. https:// doi.org/10.1016/j.tsf.2004.01.052

Lu N, Suo Z, Vlassak JJ (2010) The effect of film thickness on the failure strain of polymer-supported metal films. Acta 
Mater 58(5):1679-1687. https://doi.org/10.1016/j.actamat. 2009.11.010

Miehe C, Schotte J, Schröder J (1999) Computational micromacro transitions and overall moduli in the analysis of polycrystals at large strains. Comput Mater Sci 16(1):372-382. https://doi.org/10.1016/S0927-0256(99)00080-4

Moser S, Zernatto G, Kleinbichler M, Nelhiebel M, Zechner J, Cordill MJ, Pippan R (2019) A novel setup for in situ monitoring of thermomechanically cycled thin film metallizations. JOM 71:3399-3406. https://doi.org/10.1007/ s11837-019-03695-2

Özdemir I, Brekelmans WAM, Geers MGD (2008) FE 2 computational homogenization for the thermo-mechanical analysis of heterogeneous solids. Comput Methods Appl Mech Eng 198(3):602-613. https://doi.org/10.1016/j.cma.2008. 09.008

Renault PO, Villain P, Coupeau C, Goudeau P, Badawi KF (2003) Damage mode tensile testing of thin gold films on polyimide substrates by X-ray diffraction and atomic force microscopy. Thin Solid Films 424(2):267-273. https://doi. org/10.1016/S0040-6090(02)01127-6

Ricker S, Mergheim J, Steinmann P, Müller R (2010) A comparison of different approaches in the multi-scale computation of configurational forces. Int J Fract 166:203-214. https:// doi.org/10.1007/s10704-010-9525-2

Schröder J (2009) Derivation of the localization and homogenization conditions for electro-mechanically coupled problems. Comput Mater Sci 46(3):595-599. https://doi.org/10.1016/ j.commatsci.2009.03.035

Sengupta A, Papadopoulos P, Taylor RL (2012) A multiscale finite element method for modeling fully coupled thermomechanical problems in solids. Int J Numer Methods Eng 91(13):1386-1405. https://doi.org/10.1002/nme.4320
Sim GD, Lee YS, Lee SB, Vlassak JJ (2013) Effects of stretching and cycling on the fatigue behavior of polymer-supported Ag thin films. Mater Sci Eng A 575:86-93. https://doi.org/ 10.1016/j.msea.2013.03.043

Tada N (2006) Evaluation of the distribution of multiple circular cracks with random radii and angles by direct current electrical potential difference method. Int J Fract 141:49-62. https://doi.org/10.1007/s10704-006-7763-0

Tada N, Hayashi Y, Kitamura T, Ohtani R (1996) Analysis of direct current potential field around multiple random cracks. Int J Fract 75(1):69-84

Tada N, Hayashi Y, Kitamura T, Ohtani R (1997) Analysis on the applicability of direct current electrical potential method to the detection of damage by multiple small internal cracks. Int J Fract 85:1-9. https://doi.org/10.1023/A: 1007452331773

Temizer I (2016) Sliding friction across the scales: thermomechanical interactions and dissipation partitioning. J Mech Phys Solids 89:126-148. https://doi.org/10.1016/j.jmps. 2016.01.012

Temizer İ, Wriggers P (2011) Homogenization in finite thermoelasticity. J Mech Phys Solids 59(2):344-372. https://doi. org/10.1016/j.jmps.2010.10.004

Publisher's Note Springer Nature remains neutral with regard to jurisdictional claims in published maps and institutional affiliations. 